\title{
In vitro Chicken Bone Marrow-Derived Dendritic Cells Comprise Subsets at Different States of Maturation
}

\author{
Robin H. G. A. van den Biggelaar ${ }^{1}$, Ger J. A. Arkesteijn ${ }^{1}$, Victor P. M. G. Rutten ${ }^{1,2}$, \\ Willem van Eden ${ }^{1}$ and Christine A. Jansen ${ }^{1 *}$
}

'Department of Biomolecular Health Sciences, Division of Infectious Diseases and Immunology, Faculty of Veterinary Medicine, Utrecht University, Utrecht, Netherlands, ${ }^{2}$ Department of Veterinary Tropical Diseases, Faculty of Veterinary Science, University of Pretoria, Pretoria, South Africa

OPEN ACCESS

Edited by:

Benedicte Lambrecht, Sciensano, Belgium

Reviewed by:

Viskam Wijewardana,

International Atomic Energy

Agency, Austria

Sascha Trapp,

INRA Centre Val de Loire, France

Nandor Nagy,

Semme/weis University, Hungary

Abd Rahaman Yasmin

Putra Malaysia University, Malaysia

*Correspondence:

Christine A. Jansen c.a.jansen@uu.nl

Specialty section

This article was submitted to

Comparative Immunology,

a section of the journal

Frontiers in Immunology

Received: 09 October 2019

Accepted: 20 January 2020

Published: 26 February 2020

Citation:

van den Biggelaar RHGA,

Arkesteijn GJA, Rutten VPMG, van

Eden $W$ and Jansen CA (2020) In vitro

Chicken Bone Marrow-Derived

Dendritic Cells Comprise Subsets at

Different States of Maturation.

Front. Immunol. 11:141.

doi: 10.3389/fimmu.2020.00141
Research in chickens has been fundamental for the discovery of basic aspects of the immune system and has led to an interest in the in-depth characterization of avian immune cell types including dendritic cells (DCs). The in vitro generation and expansion of chicken bone marrow-derived DCs (chBMDCs) in the presence of granulocyte-macrophage colony-stimulating factor (GM-CSF) has provided a way to study chicken DCs, which are only present at limited cell numbers in vivo. This method has been employed to study the interactions between chicken DCs and pathogens or vaccines. However, a detailed characterization of the chBMDC culture is still lacking. In the present study, we performed an elaborate phenotypical and functional analysis of the chBMDC culture and addressed its heterogeneity. After 8 days of culture, chBMDCs comprised major histocompatibility complex class II (MHC-II) ${ }^{\text {low }}$ and $\mathrm{MHC}-\|^{\text {high }}$ subsets with different morphologies. Compared with MHC-\|low chBMDCs, the $\mathrm{MHC}$ - II high subset showed a more mature phenotype, with higher expressions of CD1.1, CD40, CD80, CCR7, and CD83, and a relatively low opsonophagocytic capacity. Nevertheless, MHC-II ${ }^{\text {high }}$ chBMDCs did not show an increased capacity to induce T-cell proliferation. Therefore, MHC-II high chBMDCs were found to be semi-mature. Interestingly, the presence of the semi-mature MHC-II ${ }^{\text {high }}$ chBMDC subset reduced when cells were cultured in the presence of IL-4. Finally, prolonged cell culture after fluorescence-activated cell sorting (FACS) converted the semi-mature MHC-II ${ }^{\text {high }}$ subset back into the immature phenotype of the $\mathrm{MHC}$ - $\|^{\text {low }}$ subset, demonstrating plasticity of their maturation state. This detailed characterization explained the heterogeneity of the chBMDC culture by the simultaneous presence of immature and semi-mature chBMDC subsets, in addition to cells without features of antigen-presenting cells. Our findings are instrumental for the interpretation of experiments using the chBMDC culture in past and future research by providing insights into its phenotypically and functionally distinct cell types.

Keywords: chicken, bone marrow-derived dendritic cells, maturation, GM-CSF, IL-4, MHC-II 


\section{INTRODUCTION}

Dendritic cells (DCs) are important innate immune cells that capture and process antigens to present them to cells of the adaptive immune system (1). Adaptive immune responses result in pathogen-specific and long-lasting immunological memory, which enables the immune system to act more rapidly upon a second encounter with the pathogen. Vaccination against pathogens critically depends on DCs, which, respectively, support and fine-tune antigen presentation by co-stimulatory molecules and cytokines. The expression pattern of costimulatory molecules and cytokines by DCs depends on signals from their environment, including pathogen-associated molecular patterns (PAMPs) $(1,2)$, damage-associated molecular patterns (DAMPs) (3), and cytokines (1, 2, 4).

DCs are rare cells in all tissues and hard to isolate, which makes the use of primary DCs in functional assays challenging. To overcome this problem, granulocyte-macrophage colony-stimulating factor (GM-CSF)-differentiated bone marrow-derived DC (BMDC) culture methods have been developed to generate DCs in large numbers (5). The availability of cultured BMDCs facilitates their use in in vitro screening methods, including immunogenicity tests for vaccines and toxicity tests for allergens (6-8). In addition to well-characterized murine BMDC culture methods, such methods have been developed for veterinary species, including dogs [Ricklin (9)], cats (10), cattle (11), sheep (12), pigs (13), and chickens (14).

Chicken BMDCs (chBMDCs) have been cultured in the presence of both recombinant chicken GM-CSF and interleukin4 (IL-4) and were defined as DCs because of their typical stellate morphology and high expression of both major histocompatibility complex class II (MHC-II) and CD11b/c (14). This chBMDC culture method has led to several studies into the role of chicken DCs in infection and vaccination. Maturation of chBMDCs has been observed after stimulation with lipopolysaccharide (LPS) or CD40L, as demonstrated by increased surface expression of co-stimulatory molecules CD40, CD83, and CD86; reduced phagocytosis and endocytosis; and an increased ability to induce a mixed lymphocyte reaction (14). Similarly, chBMDCs have been found to mature upon exposure to avian influenza virus $(15,16)$, infectious bursal disease virus (17), or Salmonella enteritidis and Salmonella gallinarum vaccine candidates $(18,19)$.

Despite the widespread use of BMDCs originating from chickens and other species, a recent transcriptome study showed that murine GM-CSF-differentiated BMDCs differ phenotypically from murine DC populations in vivo (20). Moreover, this study revealed that murine BMDC cultures comprise both $\mathrm{CD}_{11} \mathrm{~b}^{\text {high }}$ MHC-II ${ }^{\text {low }}$ macrophage-like and CD11b ${ }^{\text {low }}$ MHC-II ${ }^{\text {high }}$ DC-like subsets that are closely related, but still phenotypically and functionally different. These findings had implications for conclusions drawn using in vitro murine BMDC cultures as a model for DC biology in vivo and are part of the ongoing discussion on how to distinguish DCs and macrophages (20-25). In addition, these findings stressed the importance of thorough characterization of the cellular subsets present in in vitro BMDC cultures and triggered us to explore in depth the nature of chBMDCs raised in vitro with GM-CSF and to determine whether these indeed represent DC-like cells.

The initial results of the present study showed that the chBMDC culture was heterogeneous and comprised MHC-II ${ }^{\text {low }}$ and MHC-II ${ }^{\text {high }}$ subsets, similar to observations in murine BMDC cultures. Therefore, we hypothesized that chBMDC culture comprised MHC-II ${ }^{\text {low }}$ macrophage-like and MHC-II ${ }^{\text {high }}$ DC-like subsets. However, in contrast to murine BMDC cultures, the MHC-II ${ }^{\text {low }}$ and MHC-II ${ }^{\text {high }}$ subsets of the chBMDC culture were found to reflect different maturation states rather than distinct cell types. MHC-II ${ }^{\text {high }}$ chBMDCs were found to exhibit increased expression of costimulatory molecules, also in the absence of stimuli. These findings on chBMDCs may have important consequences for conclusions drawn in past and future studies that make use of the chBMDC culture as a model for in vivo DC biology in chickens, in particular studies that assess chBMDC maturation.

\section{MATERIALS AND METHODS}

\section{Bone Marrow Isolation}

Eighteen-day-old embryonated NOVOgen Brown eggs were obtained from a commercial breeder (Verbeek Broederij, Zeewolde, the Netherlands). Chicken embryos were removed from the eggs and euthanized by decapitation. Next, the tibiae and femurs were collected, bone heads were removed, and bone marrow was harvested by flushing the bones with RPMI-1640 cell culture medium supplemented with GlutaMAX ${ }^{\mathrm{TM}}-\mathrm{I}$, phenol red, and HEPES (Gibco ${ }^{\mathrm{TM}}$, Life Technologies Limited, Paisley, UK) under sterile conditions using a Plastipak ${ }^{\mathrm{TM}} 10-\mathrm{ml}$ syringe with a Microlance ${ }^{\mathrm{TM}} 3$ 21-G needle (both from BD Biosciences, Pharmingen, San Diego, CA, USA). Bones and bone marrow cells were kept on ice during the whole procedure. Bone marrow cells from 200 embryos were pooled, gently squeezed through a Falcon ${ }^{\circledR} 70-\mu \mathrm{m}$ cell strainer (Corning ${ }^{\circledR}$, Corning B.V. Life Sciences, Amsterdam, the Netherlands), and stored at $-140^{\circ} \mathrm{C}$ in RPMI, 50\% chicken serum (Gibco ${ }^{\mathrm{TM}}$, Life Technologies Limited, Paisley, UK), and 10\% DMSO (Honeywell, Bucharest, Romania). This procedure resulted in batches comprising $1.3-2.3 \times 10^{9}$ bone marrow cells, which were frozen at a concentration of $2.5-5$ $\times 10^{7}$ cells per cryotube.

\section{chBMDC Culture}

As previously described by others (26), chBMDCs were cultured from isolated bone marrow cells in RPMI-1640 cell culture medium supplemented with $5 \%$ chicken serum and $50 \mathrm{U} / \mathrm{ml}$ of penicillin-streptomycin (all from $\mathrm{Gibco}^{\mathrm{TM}}$, Life Technologies Limited, Paisley, UK) in the presence of recombinant GMCSF (and IL-4) at $41^{\circ} \mathrm{C}, 5 \% \mathrm{CO}_{2}$. Recombinant GM-CSF and IL-4 were produced using COS-7 cells transfected with pCI-neo (Promega Corporation, Madison, Wisconsin, USA) expressing the relevant cytokine, which were a kind gift from P. Kaiser and L. Rothwell (Roslin Institute, Edinburgh, UK). The concentrations of the recombinant cytokines are given as a dilution of supernatant from transfected COS-7 cultures in accordance with a previous study (27). GM-CSF was used at the titrated concentration $(2 \mu \mathrm{l} / \mathrm{ml})$ that resulted in the highest 
percentage of MHC- $\mathrm{II}^{+} \mathrm{CD} 40^{+} \mathrm{CD} 80^{+}$cells. In one experiment, the chBMDC culture was supplemented with GM-CSF and titrated concentrations of IL-4. Bone marrow cells were seeded at $2.5 \times 10^{6}$ cells per milliliter in $75-\mathrm{cm}^{2}$ cell culture flasks in $15 \mathrm{ml}$ of RPMI-1640 medium per flask, in $25-\mathrm{cm}^{2}$ cell culture flasks in $5 \mathrm{ml}$ of RPMI-1640 medium per flask, in Costar ${ }^{\circledR}$ sixwell plates in $2 \mathrm{ml}$ of RPMI-1640 medium per well, or in $\operatorname{Costar}^{\circledR}$ 24-well plates in $0.5 \mathrm{ml}$ of RPMI-1640 medium per well (all from Corning ${ }^{\circledR}$, Corning B.V. Life Sciences, Amsterdam, the Netherlands) depending on the required sample size. Early in the morning at day 3 , culture medium with non-adherent cells was removed, and fresh RPMI-1640 medium with GM-CSF (and IL-4) was added. Late in the afternoon at day 4, the cultures received another volume of RPMI-1640 medium with GM-CSF (and IL-4). The morphology of chBMDCs was examined by light microscopy using an EVOS FL microscope (AMG, Mill Creek, Washington, USA). In selected experiments, chBMDC cultures were matured by $100 \mathrm{ng} / \mathrm{ml}$ of LPS O127:B8 (SigmaAldrich, Saint Louis, MO, USA) stimulation for $24 \mathrm{~h}$ at day 7. To harvest the cultures at day 8 , the medium with non-adherent cells was first collected. Subsequently, loosely adherent cells were washed and collected with Dulbecco's phosphate-buffered saline (DPBS) without calcium and magnesium $\left(\mathrm{DPBS}^{-/-}\right.$; Lonza, Basel, Switzerland). Finally, the remaining adherent cells were incubated in $\mathrm{DPBS}^{-1-}$ supplemented with $5 \mathrm{mM}$ UltraPure EDTA (Invitrogen ${ }^{\mathrm{TM}}$, Life Technologies Europe BV, Bleiswijk, the Netherlands) for $10 \mathrm{~min}$ at room temperature (RT) before being collected as well. All cell-containing fluids (cell culture medium, $\mathrm{DPBS}^{-/-}$, and $\mathrm{DPBS}^{-/-} 5 \mathrm{mM}$ EDTA) obtained during the harvest procedure were pooled for subsequent experiments.

\section{Flow Cytometry Analyses and Antibodies}

Antibodies and streptavidin conjugates used in this study are listed in Table 1. All were diluted in fluorescence-activated cell sorting (FACS) buffer, containing DPBS ${ }^{-/-}+0.5 \%$ BSA and $0.005 \% \mathrm{NaN}_{3}$ (both from Sigma-Aldrich, Saint Louis, MO, USA), which was used for staining and washing steps. Antibodies and streptavidin conjugates were used at titrated concentrations to stain $0.5-1.0 \times 10^{6}$ freshly harvested chBMDCs per $50 \mu \mathrm{l}$ for $20 \mathrm{~min}$ at $4^{\circ} \mathrm{C}$. Between staining steps, chBMDCs were washed twice with FACS buffer. To assess viability, the cells were first washed in $\mathrm{DPBS}^{-/-}$and then stained with Zombie Aqua Fixable Viability Dye (BioLegend Inc., San Diego, CA, USA) diluted in DPBS $^{-1-}$ for $20 \mathrm{~min}$ at $4^{\circ} \mathrm{C}$. From each sample, 50,000-100,000 chBMDCs were analyzed using a CytoFLEX LX flow cytometer, equipped with 375-, 405-, 488-, 561-, 638-, and 808-nm lasers (Beckman Coulter Inc., Brea, CA, USA), FlowJo Software v. 10.5 (FlowJo LCC, Ashland, OR, USA), and Prism 7 (GraphPad Software Inc., San Diego, CA, USA).

\section{Immunofluorescence Microscopy Analyses}

Ethanol-cleaned 12-mm glass coverslips (Waldemar Knittel Glasbearbeitungs $\mathrm{GmbH}$, Brunswick, Germany) were placed into the wells of a 24-well cell culture plate. Next, bone marrow cells were seeded and cultured in complete RPMI culture medium in the presence of recombinant GM-CSF at $41^{\circ} \mathrm{C}, 5 \%$ $\mathrm{CO}_{2}$. At day 8, differentiated chBMDCs on glass coverslips were either washed three times with $\mathrm{DPBS}^{-1-}$ or first stained with fluorescently labeled lectin wheat germ agglutinin (WGA)Alexa Fluor 488 (Invitrogen $^{\mathrm{TM}}$, Life Technologies Europe BV, Bleiswijk, the Netherlands). Staining with WGA-Alexa Fluor 488 was performed by washing the cells twice with cold DPBS with calcium and magnesium, followed by staining with WGAAlexa Fluor 488 diluted in DPBS with calcium and magnesium for $20 \mathrm{~min}$ at $4^{\circ} \mathrm{C}$. Fixation was performed in $\mathrm{DPBS}^{-1-}$ with $4 \%$ paraformaldehyde (Alfa Aesar, Haverhill, MA, USA) for $30 \mathrm{~min}$ at RT. Subsequently, the fixatives were quenched by washing the fixed samples three times with $\mathrm{DPBS}^{-/}$and $10 \mathrm{mM}$ glycine (Merck Millipore, Burlington, MA, USA) and blocked in blocking buffer, containing DPBS ${ }^{-/}, 0.05 \%$ Tween20 (Sigma-Aldrich, Saint Louis, MO, USA), and 2\% bovine serum albumin (Sigma-Aldrich, Saint Louis, MO, USA), overnight at $4{ }^{\circ} \mathrm{C}$. The coverslips were stained with the cells faced-down on Parafilm in $25 \mu \mathrm{l}$ of blocking buffer with primary antibodies for $2 \mathrm{~h}$ and secondary antibodies for $1 \mathrm{~h}$ at RT (antibodies are listed in Table 1). In addition, a nuclear staining was performed in $25 \mu \mathrm{l}$ of blocking buffer at $10 \mu \mathrm{g} / \mathrm{ml}$ with $4^{\prime}, 6-$ diamidino-2-phenylindole (DAPI) (Sigma-Aldrich, Saint Louis, MO, USA) for 5 min at RT. Between staining steps, the samples were washed three times with DPBS $^{-/}$with $0.05 \%$ Tween20. The last wash step was performed in distilled water, before mounting the samples on Polysine ${ }^{\circledR}$ microscope slides (Menzel Glaser GmbH \& Co KG, Braunschweig, Germany) in a FluorSave reagent (Calbiochem ${ }^{\circledR}$, Merck Millipore, Burlington, MA, USA). The samples were captured using a TCS-SPE-II confocal microscope (Leica Microsystems B.V., Amsterdam, the Netherlands) equipped with 405-, 488-, 561-, and 635-nm diode lasers and processed using Fiji software (28).

\section{Phagocytosis of IgY-Opsonized Beads by chBMDC Subsets}

Chicken serum IgY fraction (Agrisera $A B$, Vännäs, Sweden) was added at $14.4 \mathrm{mg} / \mathrm{ml}$ to $1.44 \times 10^{10}$ beads per milliliter of 1 $\mu \mathrm{m}$ crimson carboxylate-modified FluoSpheres (Invitrogen ${ }^{\mathrm{TM}}$, Life Technologies Europe BV, Bleiswijk, the Netherlands) and incubated overnight on an orbital shaker at $4^{\circ} \mathrm{C}$ to create IgYopsonized beads. The next day, the beads were washed twice and resuspended in $\mathrm{DPBS}^{-/-}$with centrifugation steps at 3,000 $g$ for $20 \mathrm{~min}$ at $4^{\circ} \mathrm{C}$ in between. To confirm IgY coupling, the beads were stained in FACS buffer with MaCh IgY-PE (SouthernBiotech, Birmingham, AL, USA) and analyzed on the CytoFLEX LX flow cytometer (data not shown). Next, the IgYcoupled beads were used in a phagocytosis assay to assess bead uptake by chBMDCs. After 8 days of culture in a 24-well plate, chBMDCs from one well were harvested and counted to determine the number of IgY-opsonized beads needed to obtain a 1:1 bead-to-cell ratio. Next, crimson beads were added to the remaining wells followed by 4 -h incubation at $41{ }^{\circ} \mathrm{C}, 5 \% \mathrm{CO}_{2}$, to allow phagocytosis by chBMDCs. Subsequently, chBMDCs were harvested and stained for flow cytometry or confocal microscopy according to the methods described above. For flow cytometry, the cells were stained for MHC-II expression and viability, using Zombie Aqua Fixable Viability Dye, and analyzed using the 
TABLE 1 | Antibodies used in this study.

\begin{tabular}{|c|c|c|c|c|c|}
\hline Antibody name & Isotype & Clone & Target & Figure & Source \\
\hline MaCh-MHC-II Alexa Fluor 488 & $\lg G 1$ & $2 \mathrm{G} 11$ & MHC-II & 1 & SouthernBiotech \\
\hline MaCh-la PE & $\lg M$ & Cia & MHC-II & $2,6,7,9$ & \\
\hline MaCh-la UNLB & $\lg M$ & Cia & MHC-II & 3 & \\
\hline $\mathrm{M} \alpha \mathrm{Ch}-\beta_{2}$-microglobulin UNLB & $\lg \mathrm{G} 1$ & F21-21 & MHC-I & 2,6 & \\
\hline MaCh-monocyte/macrophage UNLB & $\lg G 1$ & KUL01 & MRC1L-B & 2,6 & \\
\hline MaCh-c-kit BIOT & $\lg G 2 a$ & Kit2c75 & c-kit, CD117 & 2,6 & \\
\hline MaCh-CD1.1 UNLB & $\lg G 1$ & CB3 & CD1.1 & 2,6 & \\
\hline M IgM, $к$ isotype Ctrl PE & $\lg M$ & MM-30 & - & 2,6 & BioLegend \\
\hline MaCh-CD40 UNL & $\lg \mathrm{g} 2 \mathrm{a}$ & AV79 & CD40 & 2,6 & Bio-Rad \\
\hline MaCh-CD80 UNLB & $\lg G 2 a$ & IAH:F864DC7 & CD80, B7-1 & $2,3,6,7,9$ & \\
\hline MaCh-CSF1R UNLB & IgG1 & ROS-AV170 & CSF1R, CD115 & $2,3,6,7,9$ & \\
\hline MaCh-5C7 UNLB & $\lg \mathrm{G} 1$ & $5 \mathrm{C7}$ & Putative CD11b/c* & 2,6 & Produced in house $^{\dagger}$ \\
\hline RaM-IgG1 BV421 & $\lg G$ & RMG1-1 & Mouse IgG1 & 7,9 & BioLegend \\
\hline Streptavidin APC & - & - & Biotin & 2,6 & BD Pharmingen \\
\hline GaM-IgG1 APC & $\lg G$ & - & Mouse IgG1 & 2,6 & SouthernBiotech \\
\hline GaM-lgG2a APC & $\lg G$ & - & Mouse IgG2a & $2,6,7,9$ & \\
\hline GaM-IgM Alexa Fluor 488 & $\lg G$ & - & Mouse lgM & 3 & \\
\hline GaM-lgG2a Alexa Fluor 568 & $\lg G$ & - & Mouse IgG2a & 3 & Invitrogen \\
\hline GaM-IgG1 Alexa Fluor 647 & $\lg G$ & - & Mouse lgG1 & 3 & \\
\hline
\end{tabular}

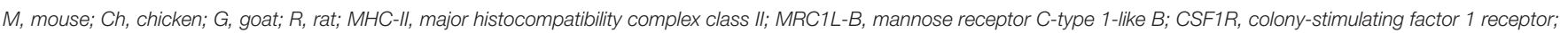

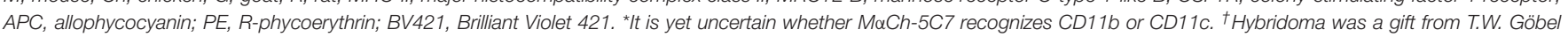

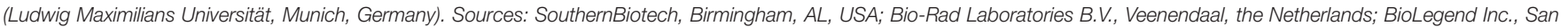
Diego, CA, USA; Invitrogent ${ }^{\dagger}$, Life Technologies Europe BV, Bleiswijk, the Netherlands; BD Biosciences, Pharmingen, San Diego, CA, USA.

CytoFLEX LX flow cytometer. For confocal microscopy, the cells were stained with WGA-Alexa Fluor 488, M $\alpha \mathrm{Ch}$-Ia BIOT, and streptavidin Alexa Fluor 405 and analyzed using the TCS-SPE-II confocal microscope.

\section{IL-4 Bioactivity Assessment by ${ }^{3} \mathrm{H}$-Thymidine Incorporation by PBMCs}

The ${ }^{3} \mathrm{H}$-thymidine incorporation assay to measure IL-4 bioactivity was modified from a published method to assess peripheral blood mononuclear cell (PBMC) proliferation (27). Heparinized blood was collected from healthy chickens (under registration number AVD108002016642-1 from the Dutch Central Authority for Scientific Procedures on Animals). The chickens were daily monitored by animal caretakers for signs and symptoms of disease, which were absent for the chickens used in this study. PBMCs were isolated from heparinized blood by density gradient separation using Ficoll-Paque PLUS (GE Healthcare, Chicago, IL, USA) according to standard procedure. Collected PBMCs were resuspended in $2 \mathrm{ml}$ of Iscove's modified Dulbecco's medium (IMDM) culture medium supplemented with GlutaMAX ${ }^{\mathrm{TM}}-\mathrm{I}$, phenol red, and HEPES, with $8 \%$ fetal bovine serum (FBS), $2 \%$ chicken serum, and $50 \mathrm{U} / \mathrm{ml}$ of penicillin-streptomycin (all from Gibco ${ }^{\mathrm{TM}}$, Life Technologies Limited, Paisley, UK). The cells were counted and seeded in a 96-well flat-bottom culture plate with $100 \mu \mathrm{l}$ of IMDM medium per well containing $2 \times 10^{5}$ cells. The cells received different concentrations (ranging 1:25-1:250) of cell culture supernatant from COS-7 cells transfected with an IL- 4 or empty pCI vector and were incubated for 4 days at $41^{\circ} \mathrm{C}, 5 \% \mathrm{CO}_{2}$. Subsequently, 0.4 $\mu \mathrm{Ci}$ of ${ }^{3} \mathrm{H}$-thymidine per well was added to the culture for $18 \mathrm{~h}$, and the cells were harvested using a Harvester 96 (TOMTEC Imaging Systems $\mathrm{GmbH}$, Unterschleißheim, Germany). ${ }^{3} \mathrm{H}-$ Thymidine incorporation by the cells was determined in a 1,450 MicroBeta Plus liquid scintillation counter (Wallac, PerkinElmer Life Sciences, Zaventem, Belgium).

\section{Separation of chBMDC Subsets by FACS}

For sorting, chBMDCs were stained with antibodies specific for MHC-II, colony-stimulating factor 1 receptor (CSF1R), and CD80 as before. In addition, the dye 7 -aminoactinomycin D (7-AAD; BD Biosciences, Pharmingen, San Diego, CA, USA) was added to the cells for viability assessment. Next, the cells were resuspended in $\mathrm{DPBS}^{-/-}, 1 \% \mathrm{FBS}, 2 \mathrm{mM}$ EDTA, and $0.005 \% \mathrm{NaN}_{3}$ and flushed through a $70-\mu \mathrm{m}$ cell strainer to create single-cell suspensions. MHC-II ${ }^{\text {high }} \mathrm{CSF}^{\mathrm{R}}{ }^{\text {low }}$ and MHC-II ${ }^{\text {low }}$ CSF1R $R^{\text {high }}$ chBMDC subsets were sorted by FACS with a BD Influx cell sorter, equipped with 405-, 488-, 561-, and 635-nm lasers (BD Biosciences, Pharmingen, San Diego, CA, USA). Each chBMDC subset constituted close to $25 \%$ of the original sample. Approximately $2 \times 10^{6}$ cells were sorted for both subsets to perform quantitative real-time PCR (RT-qPCR). In parallel, the cells were analyzed before and after FACS using the CytoFLEX LX flow cytometer. Moreover, some cells were sorted onto a Polysine ${ }^{\circledR}$ microscope slide, fixed with $4 \%$ paraformaldehyde, and analyzed by confocal microscopy using the TCS-SPE-II microscope to confirm the expression patterns of MHC-II, CD80, and CSF1R by the chBMDC subsets. In addition, chBMDCs were sorted to evaluate the phenotypic stability of the cells by 
TABLE 2 | All primer and probe sequences are given from the $5^{\prime}$ to $3^{\prime}$ ends.

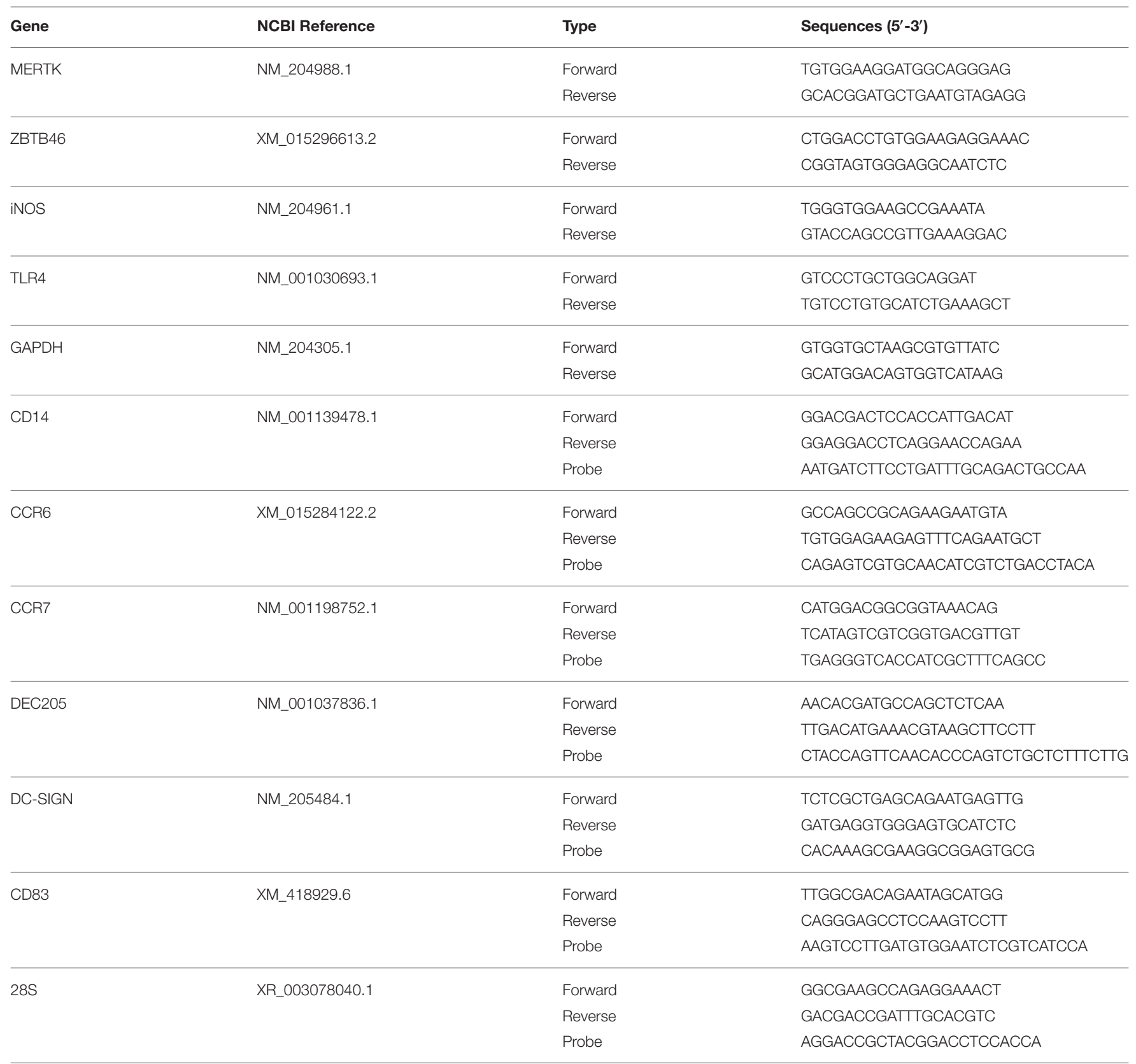

In the absence of probe sequences, quantitative real-time PCR (RT-qPCR) was performed with SYBR Green Master Mix.

prolonged cell culture. Sorted chBMDCs were seeded into 24well plates at 350,000 cells per well in $1 \mathrm{ml}$ of RPMI culture medium with GM-CSF and incubation for 1 or 3 days of prolonged cell culture at $41^{\circ} \mathrm{C}, 5 \% \mathrm{CO}_{2}$, before repeated flow cytometric analysis.

\section{Gene Expression Analysis of Separated chBMDC Subsets Using RT-qPCR}

Sorted chBMDC subsets were lysed in RLT buffer (Qiagen $\mathrm{GmbH}$, Hilden, Deutschland) and stored at $-20^{\circ} \mathrm{C}$ until RNA isolation. RNA isolation was performed with the RNeasy Mini Kit (Qiagen GmbH, Hilden, Deutschland) according to the manufacturer's instructions, including a DNase treatment step using the RNase-Free DNase Set (Qiagen GmbH, Hilden, Deutschland). Next, cDNA was prepared using the reverse transcriptase from the iScript cDNA Synthesis Kit (Bio-Rad Laboratories B.V., Veenendaal, the Netherlands) according to the manufacturer's instructions. RT-qPCRs were performed with primers (listed in Table 2) and either FAM-TAMRA-labeled TaqMan probes combined with TaqMan Universal PCR Master Mix or SYBR Green Master Mix without probes (all from Life 
Technologies Europe BV, Bleiswijk, the Netherlands). RT-qPCRs were performed with a CFX Connect and analyzed with the CFX Maestro software (both from Bio-Rad Laboratories B.V., Veenendaal, the Netherlands). All RT-qPCRs were evaluated for proper amplification efficiency (95-105\%) using serial dilutions of reference cDNA from splenocytes that were stimulated with concanavalin A for $24 \mathrm{~h}$ or from HD11 cells that were stimulated with LPS for $3 \mathrm{~h}$. RT-qPCRs were performed in triplo for every sample, and the average gene expression levels were expressed as 40-Ct values, as described by Eldaghayes et al. (29). The results were normalized toward gene expression levels of the housekeeping genes $28 S$ and GAPDH.

\section{Mixed Lymphocyte Reaction Between chBMDC Subsets and PBMCs}

PBMCs were isolated from heparinized blood by density gradient separation using Ficoll-Paque PLUS according to standard procedure and resuspended in X-VIVO 15 cell culture medium. The cells were seeded in a 96-well flat-bottom culture plate with 100,000 cells per well. Unsorted and sorted MHC-II low and MHC-II ${ }^{\text {high }}$ chBMDC subsets were resuspended in X-VIVO 15 medium and added to the PBMCs at different effector-to-target (E:T) ratios, ranging 1:2-1:8. A positive control was created by addition of $1 \mu \mathrm{g} / \mathrm{ml}$ of anti-CD3, $1 \mu \mathrm{g} / \mathrm{ml}$ of anti-CD28, and 1:50 supernatant from a COS-7 cells transfected with a pCIneo construct expressing chicken IL-2. A negative control was created by adding an additional X-VIVO 15 medium. The cells were cultured for 3 days at $41^{\circ} \mathrm{C}, 5 \% \mathrm{CO}_{2}$. Subsequently, 0.4 $\mu \mathrm{Ci}$ of ${ }^{3} \mathrm{H}$-thymidine per well was added to the culture for $18 \mathrm{~h}$, and the cells were harvested using a Harvester. ${ }^{3} \mathrm{H}$-Thymidine incorporation by the cells was determined in a 1,450 MicroBeta Plus liquid scintillation counter.

\section{Statistical Analysis}

Statistical analysis was performed using GraphPad Prism 7 software (GraphPad Software, La Jolla, CA, USA). The data were tested for the assumptions of normally distributed data. Flow cytometry expression data showing the geometric mean fluorescent intensity (gMFI) was log-transformed in order to generate normally distributed data. Paired $t$-tests were used to test for statistically significant differences between MHC-II ${ }^{\text {low }}$ and MHC-II ${ }^{\text {high }}$ chBMDC subsets. A $p$-value of $<0.05$ was considered statistically significant.

\section{RESULTS}

\section{chBMDC Cultures Are Heterogeneous and Comprise MHC-II low and MHC-II high Subsets Cells That Differ in Morphology and Phenotype}

Bone marrow cells, derived from bones of 18-day embryonated chicken embryos, were cultured in the presence of GM-CSF for 7 days to generate chBMDCs. The bone marrow isolates comprised mainly myeloid immune cells, including monocytes, thrombocytes, thromboblasts, granulocytes, and erythrocytes (Supplementary Figure 1A). After 7 days of culturing, nearly all cells were found to be $\mathrm{CD} 45^{+}$and thus hematopoietic (Supplementary Figure 1B). In agreement with previous studies, chBMDCs formed clusters holding veiled cells (Figures 1A,C, arrows), typical for DC morphology (14), and highly expressing MHC-II (Figure 1B). MHC-II-expressing cell clusters did not appear in the absence of GM-CSF (Supplementary Figure 2). Beyond the clusters, cells with different morphologies were visible. These were large, round, without protrusions, and highly granular (Figure 1A, stars). Upon LPS stimulation, cell clusters became less dense, and many individual small cells with elongated protrusions became visible, a feature typical for mature DCs (Figure 1D, arrows).

Next, a phenotypic analysis of the chBMDC culture by flow cytometry identified three subsets, distinguished by forward scatter (FSC) vs. MHC-II expression: FSC ${ }^{\text {low }}$ with no or low expression of MHC-II (FSC ${ }^{\text {low }}$ ), FSC ${ }^{\text {int }}$ with high expression of MHC-II (MHC-II ${ }^{\text {high }}$ ), and FSC ${ }^{\text {high }}$ with low expression of MHC-II (MHC-II ${ }^{\text {low }}$ ) (Figure 2A). These chBMDC subsets were evaluated for the expression of myeloid markers including integrin $\mathrm{CD} 11 \mathrm{~b} / \mathrm{c}$, costimulatory receptors CD40 and CD80, CSF1R, stem cell growth factor receptor c-kit, mannose receptor C-type 1-like B (MRC1L-B), non-classical MHC molecule CD1.1, and MHC class I component $\beta_{2}$-microglobulin $(\beta 2 \mathrm{~m})$. FSC ${ }^{\text {low }}$ cells showed high expression of $\mathrm{CD} 1 \mathrm{~b} / \mathrm{c}$, but no expression of CD40 and CD1.1 (Figure 2B). CD80, c-kit, MRC1L-B, and $\beta 2 \mathrm{~m}$ were expressed at moderate levels. CSF1R and c-kit were expressed by some FSClow cells, but not by others, showing further heterogeneity within this subset. Since FSC ${ }^{\text {low }}$ cells were largely positive for MRC1L-B but showed no or low expression of $\mathrm{MHC}$ molecules and costimulatory molecules, these were likely to represent undifferentiated monocytes. Both MHC-II high and $\mathrm{MHC}-\mathrm{II}^{\text {low }}$ cells showed expression of MHC molecules and costimulatory molecules. Compared to MHC-II ${ }^{\text {low }}$ cells, MHCII $^{\text {high }}$ cells expressed higher levels of costimulatory receptors CD40 and CD80, CD1.1, and MRC1L-B (Figures 2B,C and Supplementary Table 1). Conversely, MHC-II ${ }^{\text {low }}$ cells expressed higher levels of $\mathrm{CD} 11 \mathrm{~b} / \mathrm{c}, \mathrm{CSF} 1 \mathrm{R}, \mathrm{c}-\mathrm{kit}$, and $\beta 2 \mathrm{~m}$. Taken together, MHC-II low and MHC-II ${ }^{\text {high }}$ chBMDC subsets both showed a phenotype of antigen-presenting cells, but differentially expressed many myeloid markers.

Next, the expression patterns of CSF1R, MRC1L-B, and CD80 by MHC-II ${ }^{\text {low }}$ and MHC-II ${ }^{\text {high }}$ chBMDC subsets were evaluated by immunofluorescent confocal microscopy. The MHC-II ${ }^{\text {low }}$ subset expressed higher levels of CSF1R than the MHC-II ${ }^{\text {high }}$ subset (Figure 3A), in accordance with the flow cytometry data (Figure 2C). Therefore, CSF1R could be used as an additional marker to discriminate between chBMDC subsets. MHC-II ${ }^{\text {low }}$ CSF1 $\mathrm{R}^{\text {high }}$ cells were found to be large and round and to have few protrusions, indicative of a macrophage-like morphology. Similar cells were observed by light microscopy (Figure 1A, stars). In contrast, MHC-II high $\mathrm{CSF}^{\text {low }}{ }^{\text {cells }}$ showed irregular shapes with many protrusions, indicative of a DC-like morphology, and resemble the veiled cells that were observed by light microscopy (Figure 1A). MRC1L-B and CD80 expression levels were found to be highest on MHCII $^{\text {high }}$ chBMDCs (Figures 3B,C), in accordance with the flow cytometry data (Figure 2C). Nevertheless, MRC1L-B and CD80 


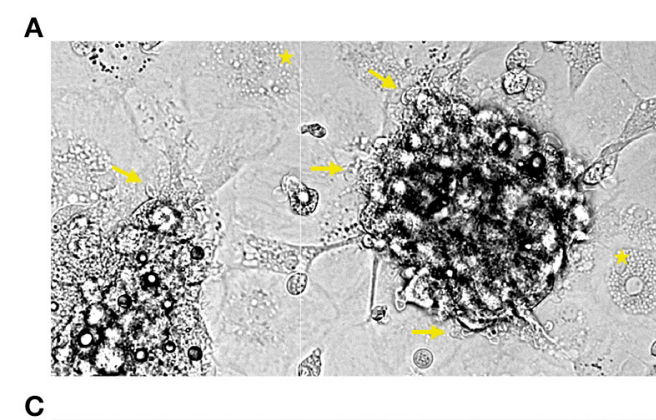

C

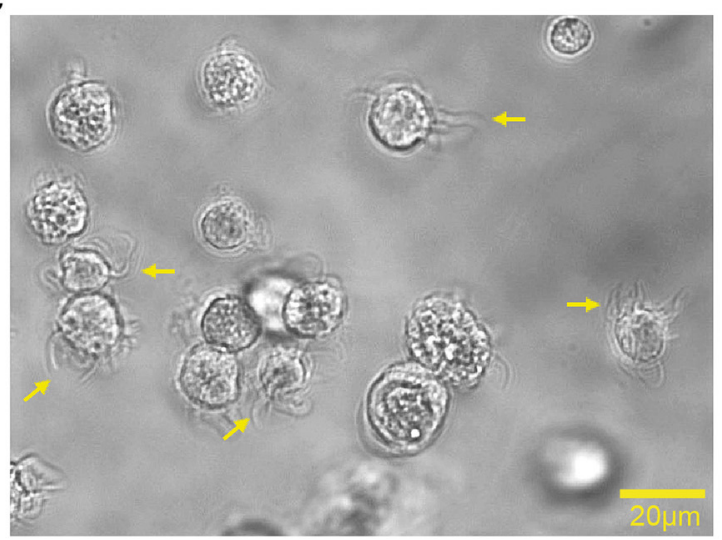

B

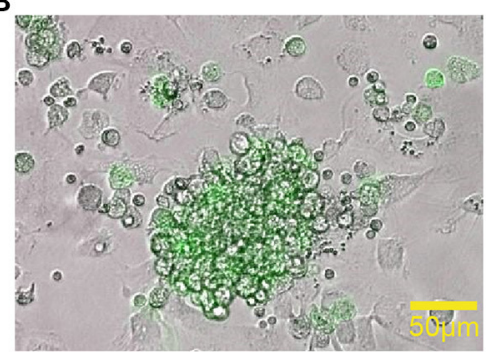

D

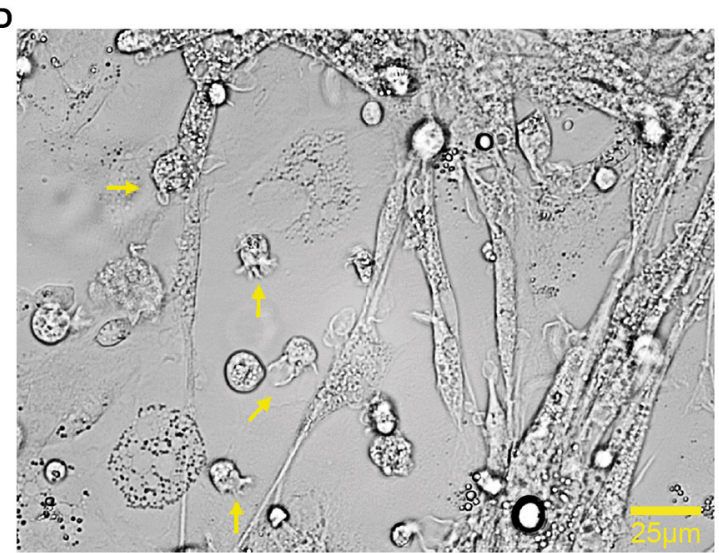

FIGURE 1 | Chicken bone marrow-derived dendritic cell (chBMDC) cultures contained clusters of major histocompatibility complex class II (MHC-II)+ cells. (A) Bright-field light microscopy shows unstimulated chBMDCs after 7 days of culture. A cluster with chBMDCs is visible at the center. Yellow arrows indicate dendritic cells, visible as veiled cells that show protrusions. Yellow stars indicate highly granular cells without protrusions. (B) An overlay of light and fluorescent microscopy shows a cluster of unstimulated chBMDCs with MHC-II expression in green. (C) Light microscopy shows individual unstimulated chBMDCs that were transferred to a Petri dish after gentle resuspension in DPBS ${ }^{-1-}$. (D) Light microscopy shows lipopolysaccharide (LPS)-stimulated chBMDCs.

did not colocalize with MHC-II. MRC1L-B and CD80 were mainly found in intracellular compartments, whereas MHC-II was found more on the cellular surface of MHC-II ${ }^{\text {high }}$-expressing chBMDCs. A stronger colocalization was observed between MRC1L-B and CD80 (Figure 3D).

\section{MHC-IIlow chBMDCs Have a Higher Capacity to Phagocytose Fluorescent Beads Compared to MHC-II ${ }^{\text {high }}$ Cells}

To assess whether the phenotypic distinction between MHCII $^{\text {low }}$ and MHC-II high chBMDCs was functionally relevant, the subsets were assessed for their ability to phagocytose chicken IgY-coated fluorescent latex beads. First, the uptake of IgYcoated crimson fluorescent beads by chBMDCs was confirmed by showing that the beads localize beneath the surface of the plasma membrane, which was visualized using WGA (Figure 4A and Supplementary Video 1). chBMDCs were stained for MHCII to identify the MHC-II high subpopulation. Both MHC-II ${ }^{\text {low }}$ and MHC-II high chBMDCs were found to take up beads as determined by confocal immunofluorescent microscopy. Next, the bead content of the chBMDC subsets was quantified by flow cytometry (Figure 4B). On average, MHC-II ${ }^{\text {low }}$ cells $(0.54$ beads per cell) contained 2.4 times more beads than MHC-II ${ }^{\text {high }}$ cells ( 0.23 beads per cell), which shows that the MHC-II ${ }^{\text {low }}$ and MHCII $^{\text {high }}$ chBMDC subsets differ in opsonophagocytic capacity
(Figure 4C). A major part of IgY beads actually bound to the cells instead of being taken up, as shown in a separate experiment performed at $4^{\circ} \mathrm{C}$ (Supplementary Figure 3). However, when bound $\mathrm{IgY}$ beads from the experiment performed at $4^{\circ} \mathrm{C}$ were subtracted from the experiment performed at $41^{\circ} \mathrm{C}, \mathrm{MHC}-\mathrm{II}^{\text {low }}$ cells were still found to take up 2.7 times more beads than MHCII $^{\text {high }}$ cells. The FSC ${ }^{\text {low }}$ chBMDC subset showed little uptake of beads ( 0.048 beads per cell) (Figure 4B).

\section{Addition of Recombinant IL-4 to chBMDC Cultures Leads to a Smaller Proportion of MHC-II ${ }^{\text {high }}$ Cells}

The effect of recombinant IL-4 on the generation of chBMDC subsets was investigated, since this cytokine has been used to generate chBMDCs by others (14). To confirm that recombinant IL-4, produced in COS-7 cells, was biologically active, its ability to induce PBMC proliferation was demonstrated (Supplementary Figure 4). Next, IL-4 was given to chBMDCs alone or in combination with GM-CSF. IL-4 alone led to few MHC-II-expressing clusters of chBMDCs (Supplementary Figure 2). IL-4 in combination with GM-CSF led to many MHC-II-expressing clusters, similar to GM-CSF alone. Next, the proportion of $\mathrm{MHC}-\mathrm{II}^{\text {low }}$ and $\mathrm{MHC}-\mathrm{II}^{\text {high }}$ cells was quantified by flow cytometry. The addition of IL-4 to the standard chBMDC culture with GM-CSF was found to 


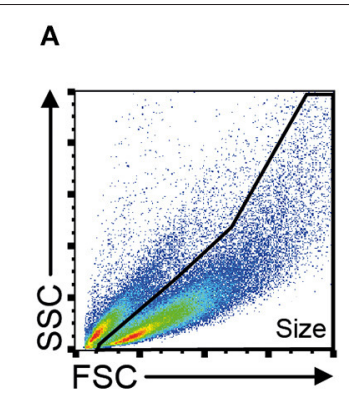

B
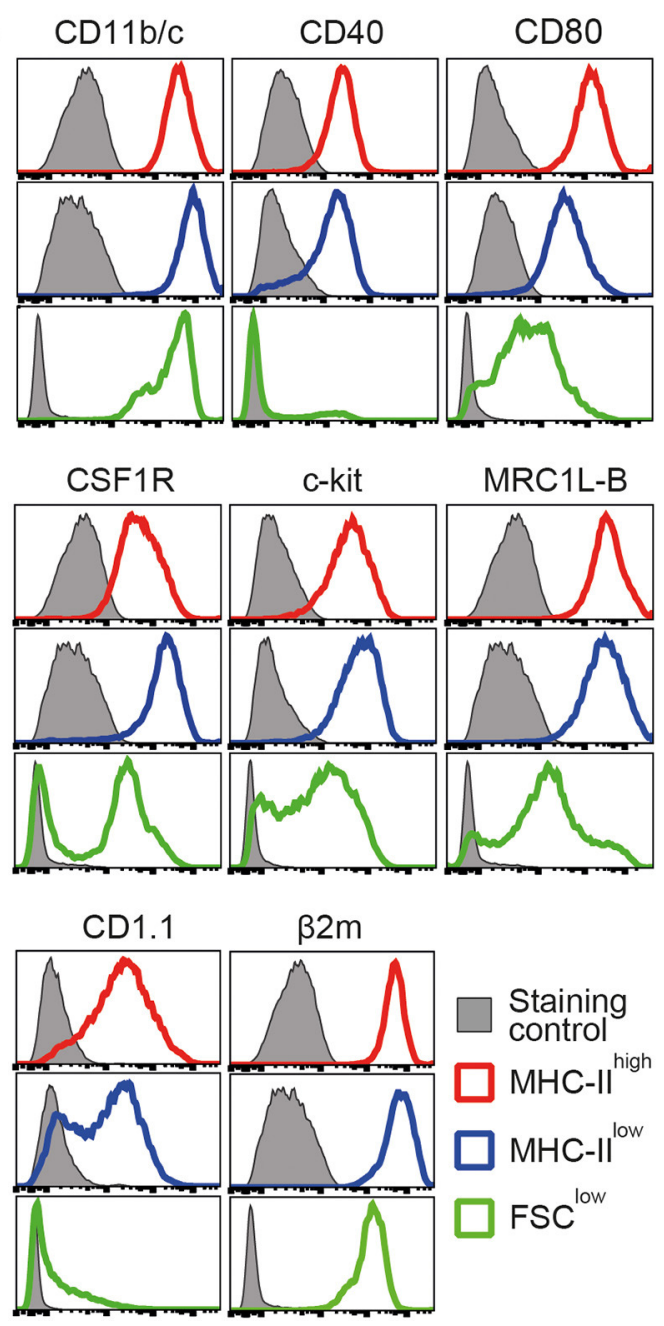
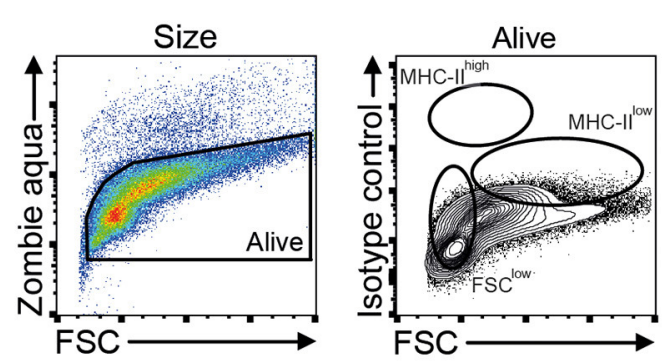

C $\quad \mathrm{CD} 11 \mathrm{~b} / \mathrm{c}$

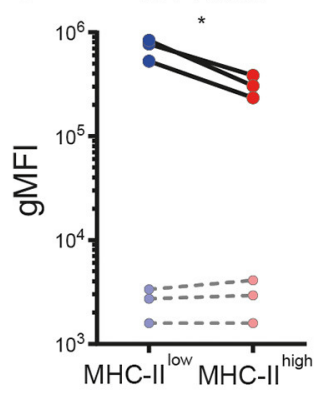

CSF1R

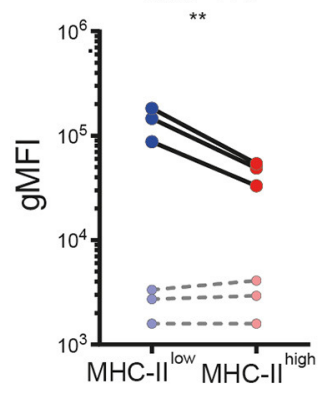

CD1.1

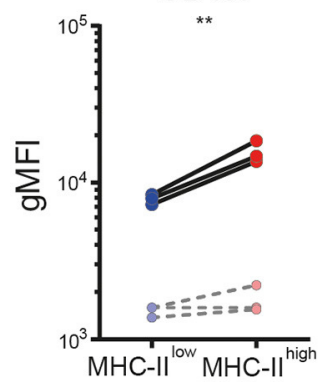

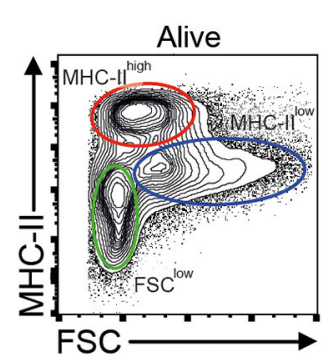
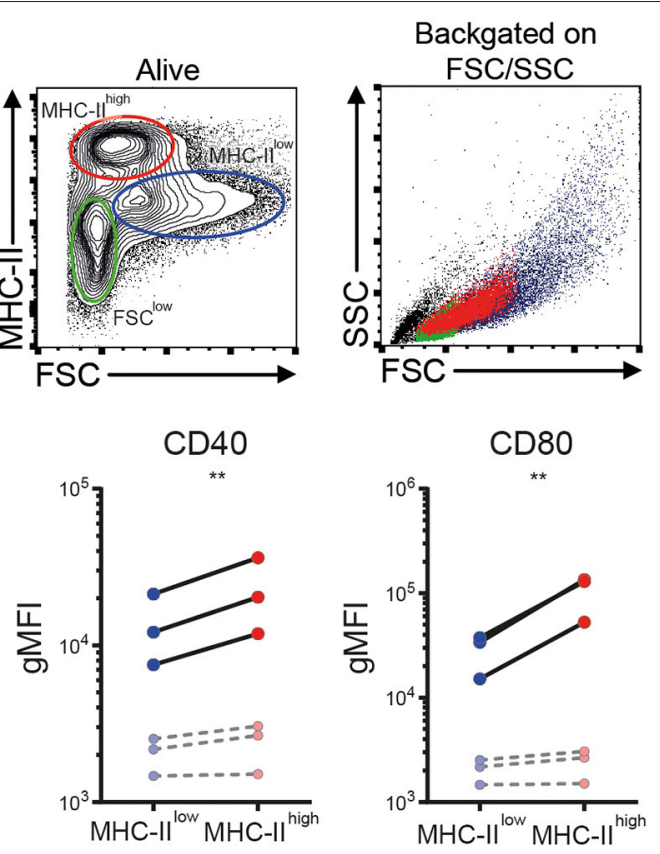

c-kit
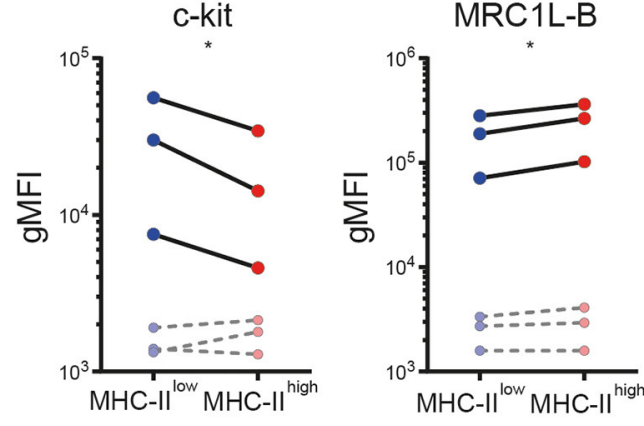

$\beta 2 \mathrm{~m}$
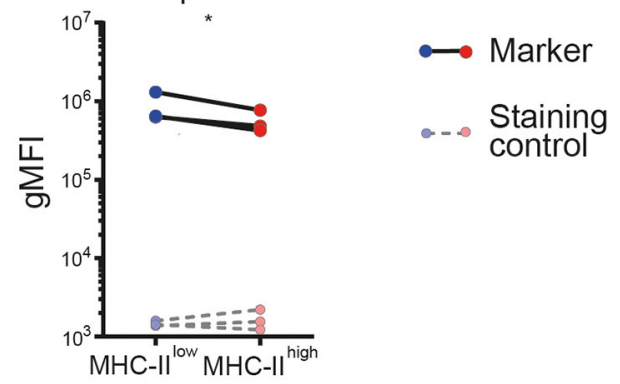

FIGURE 2 | Phenotypic analysis of chicken bone marrow-derived dendritic cells (chBMDCs) shows the presence of different subpopulations. (A) The cells were gated, respectively, for their scatter profile [forward scatter (FSC)/side scatter (SSC)], viability (Zombie Aqua), and expression of major histocompatibility complex class II (MHC-II), as shown in the first, second, and fourth panels. The third panel shows the signal after staining the cells with an isotype control antibody that corresponds

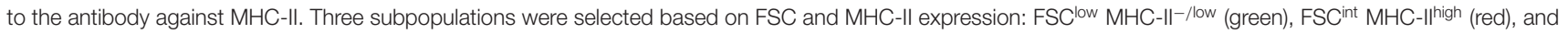
FSChigh MHC-II low (blue). The last panel shows the scatter profiles of the different subsets in matched colors, which were overlaid on the first panel. (B) Expression levels of a set of phenotypic markers are shown by representative histograms for the three subpopulations in corresponding colors. Each marker addressed was stained with a combination of either unconjugated primary antibody and allophycocyanin (APC)-conjugated secondary antibody or biotinylated primary antibody and APC-conjugated streptavidin. Filled gray histograms represent cells that have been stained with APC-conjugated streptavidin or secondary antibody to show the background fluorescence. (C) For the $\mathrm{MHC}$ - Il ${ }^{\text {low }}$ and $\mathrm{MHC}$ - Il ${ }^{\text {high }}$ subpopulations, the expression levels of all phenotypic markers are expressed as the geometric mean fluorescent intensity (gMFI) for three independent replicates. The gray dashed lines represent corresponding controls that have been stained with APC-conjugated streptavidin or secondary antibody alone. Statistically significant differences between chBMDC subsets are shown/indicated by ${ }^{*} p<0.05$ and ${ }^{* *} p<0.01$. 


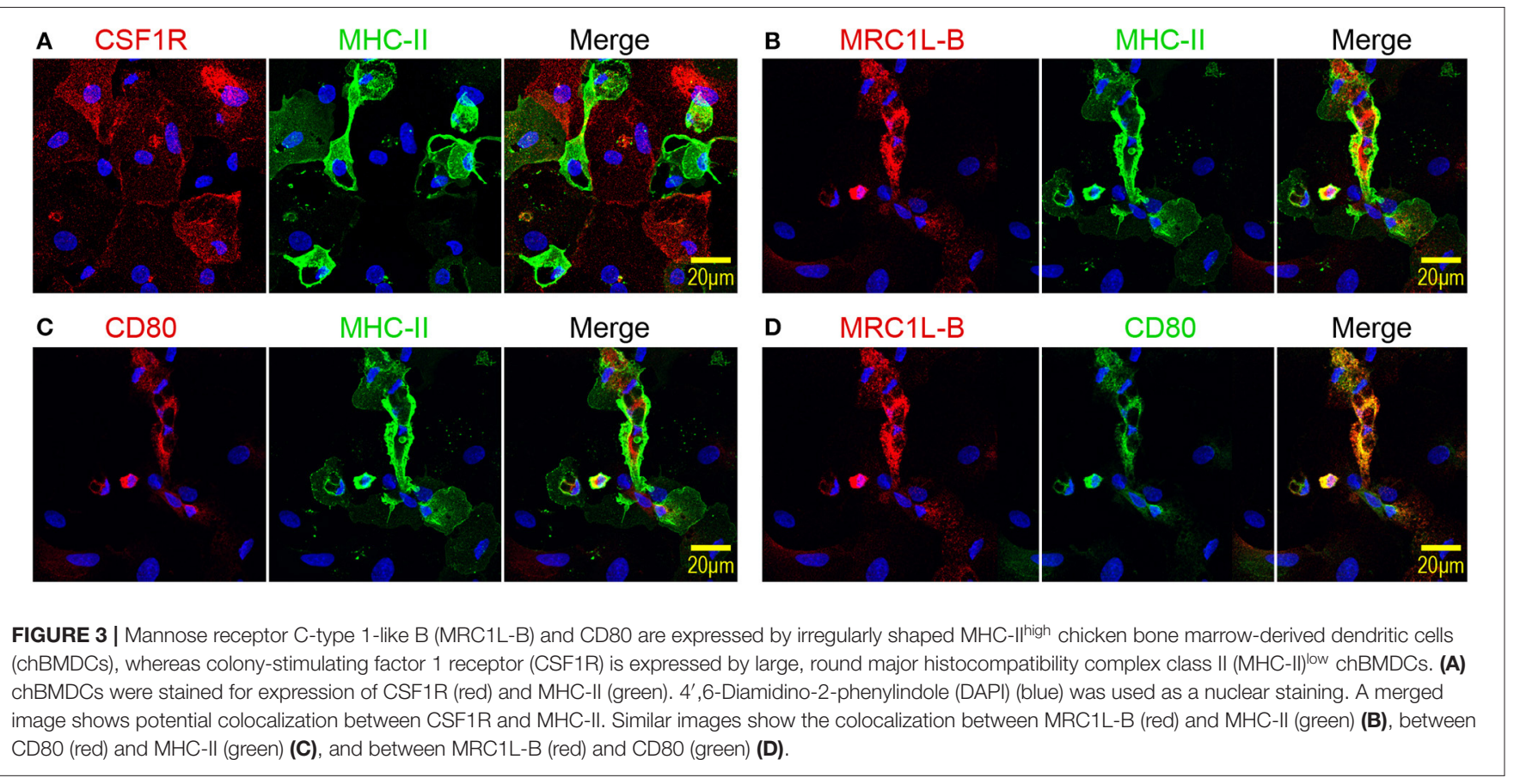

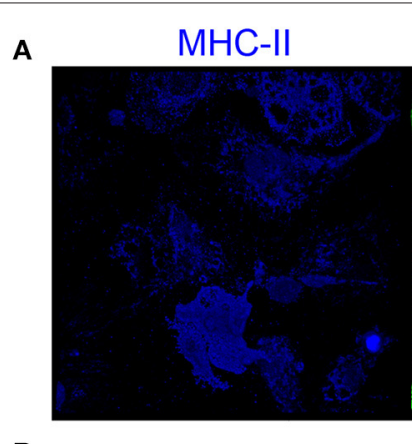

B

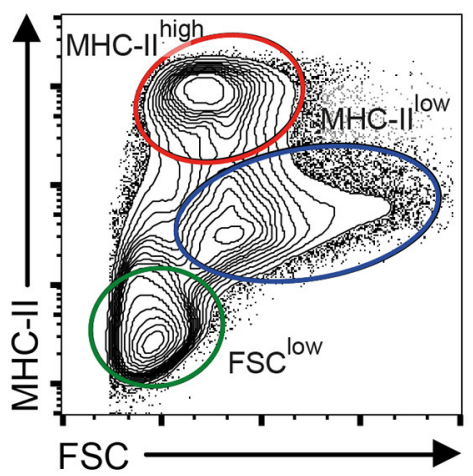

WGA
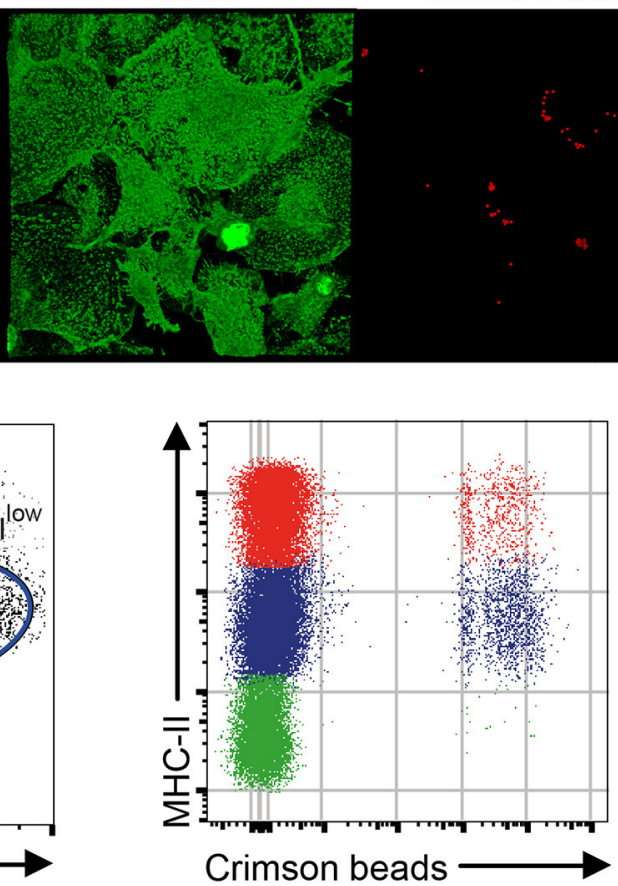

Overlay

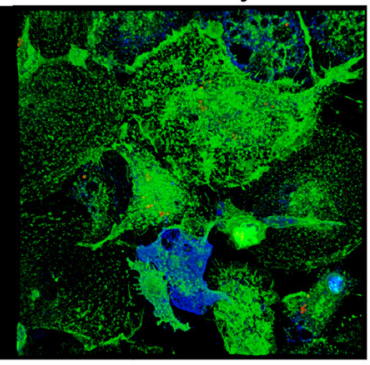

C

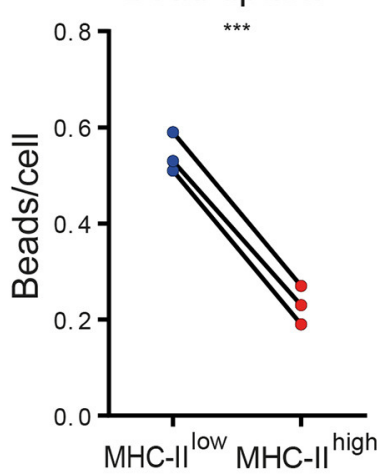

FIGURE 4 | Major histocompatibility complex class II (MHC-II) ${ }^{\text {low }}$ chicken bone marrow-derived dendritic cells (chBMDCs) phagocytose lgY-coated beads more efficiently than MHC-IIigh chBMDCs. (A) chBMDCs were stained with wheat germ agglutinin (WGA) to visualize the plasma membrane, shown in green, and MHC-II to identify MHC-II high cells, shown in blue, by confocal microscopy. Fluorescent crimson beads are shown in red. A 3D image was constructed from 56 z-stacks over a total distance of $9.4 \mu \mathrm{m}$. Here, a top view on the 3D image is shown, whereas all dimensions are visible in Supplementary Video 1. (B) Next, the bead content was quantified by flow cytometry for the previously identified chBMDC subpopulations. (C) The average number of beads per cell was calculated for MHC-Ilow and MHC-I ${ }^{\text {high }}$ subpopulations by dividing the mean fluorescent intensity (MFI) of the fluorescent beads by the MFI of cells that contained one bead (visible in $\mathbf{B}$ as the first positive line of cells). Each line represents an independent replicate $(n=3)$. A statistically significant difference between chBMDC subsets is shown/indicated by ${ }^{* \star *} p<0.001$. 

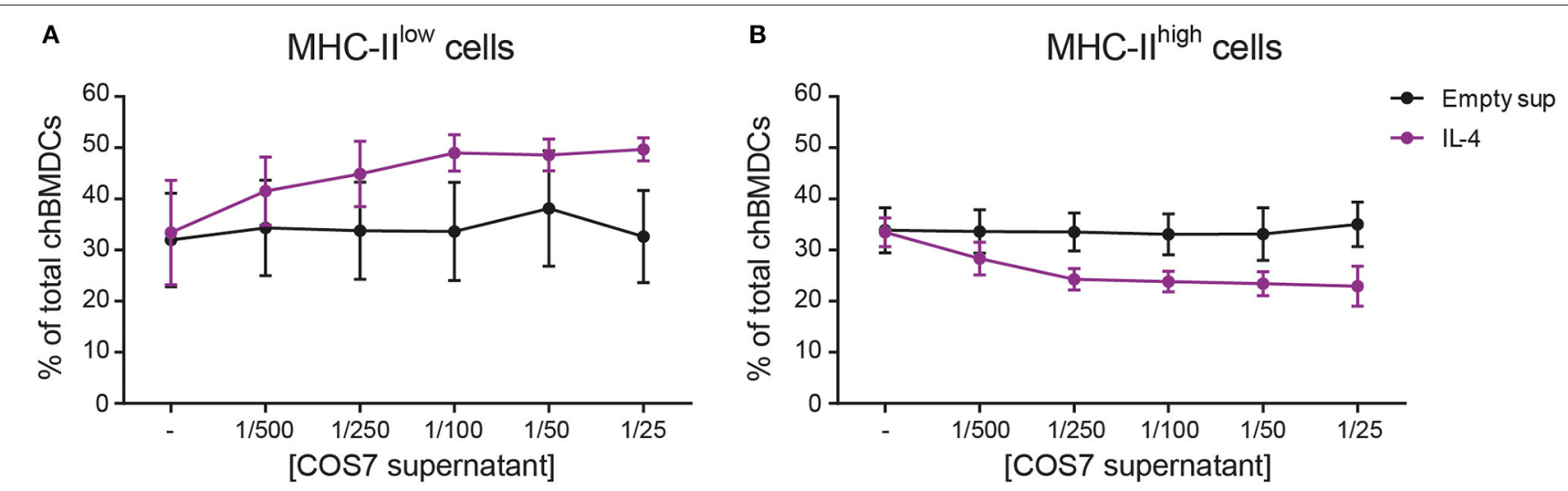

FIGURE 5 | Addition of interleukin-4 (IL-4) to the culture medium during chicken bone marrow-derived dendritic cell (chBMDC) culture reduces the proportion of major histocompatibility complex class II (MHC-II) high cells. The effect of IL-4 on MHC-Illow (A) and MHC-II high chBMDC (B) subsets was assessed by flow cytometry. Supernatant from a COS-7 cell culture transfected with recombinant IL-4 (purple) was used as a source of IL-4, and supernatant from COS-7 cells transfected with an empty vector was used as a control (black). The graphs show the percentages of both chBMDC subsets within total chBMDCs after culturing the cells in the presence of titrated concentrations of IL-4 or empty supernatant control. Both panels show the mean of four independent replicates; the error bars of all panels show the SEM.

increase the proportion of the MHC-II ${ }^{\text {low }}$ from 32.6 to $49.7 \%$ at the highest administered dose (1/25 dilution) (Figure 5A). The increase in the proportion of MHC-II ${ }^{\text {low }}$ cells occurred largely at the expense of the MHC-II ${ }^{\text {high }}$ subset, which changed proportionally from 35.0 to $23.0 \%$ at the highest administered dose of IL-4 (Figure 5B). The proportion of FSC $^{\text {low }}$ cells remained fairly stable (data not shown).

\section{LPS Stimulation Affects the Difference in Phenotype Between MHC-II low and MHC-II high chBMDCs}

To determine the effect of LPS, commonly used to induce BMDC maturation, the cells cultured for 7 days were stimulated with $100 \mathrm{ng} / \mathrm{ml}$ of LPS for $24 \mathrm{~h}$. MHC-II low and MHC-II ${ }^{\text {high }}$ chBMDC subsets were still detected (Figure 6A). Both MHC$\mathrm{II}^{\text {low }}$ and MHC-II high $c h B M D C s$ upregulated CD40, CD1.1, and $\beta 2 \mathrm{~m}$ expression, whereas the subsets downregulated c-kit and MRC1L-B expression (Figures 6B,C). CD11b/c, CSF1R, and ckit expression on MHC-II low cells decreased to levels similar to those on the MHC-II ${ }^{\text {high }}$ subset, while CD80 expression on MHC-II ${ }^{\text {high }}$ cells decreased to a level similar to that on the MHC-II ${ }^{\text {low }}$ subset. These expression patterns suggest that the phenotypes of the MHC-II ${ }^{\text {low }}$ and MHC-II ${ }^{\text {high }}$ subsets partially converged. Convergence in expression levels was also observed for CD40 and $\beta 2 \mathrm{~m}$, but not for MRC1L-B and CD1.1.

\section{chBMDC Subsets Differ in Maturation Status Rather Than Cell Type}

MHC-II ${ }^{\text {low }}$ and MHC-II ${ }^{\text {high }}$ chBMDC subsets were sorted by FACS to determine the differential expression of macrophageand DC-related genes by RT-qPCR, since well-characterized monoclonal antibodies for these cell surface markers in chickens are scarce. Since MHC-II and CSF1R showed good discrimination between the subsets in confocal microscopy (Figure 3), these markers were used to separate the subsets by FACS (Figure 7A). In addition, chBMDCs were stained for $\mathrm{CD} 80$, which was found to be more highly expressed by the MHC-II ${ }^{\text {high }}$ CSF1R ${ }^{\text {low }}$ subset than the MHC-II low CSF1R ${ }^{\text {high }}$ subset (Figure 7A), in accordance with previous results (Figure 2C). Both subsets were sorted to above $90 \%$ purity as determined by flow cytometric reanalysis after each sort (Figure 7B). Sorted cells were analyzed by fluorescent microscopy to confirm surface expression patterns of MHCII, CSF1R, and CD80 (Figure 7C). In accordance with the flow cytometry data, MHC-II and CD80 were expressed by the MHC-II ${ }^{\text {high }}$ CSF1R ${ }^{\text {low }}$ sorted subset, but not by the MHCII $^{\text {high }}$ CSF1R ${ }^{\text {low }}$ subset. In contrast, CSF1R was shown to be present on both sorted subsets. Next, RNA was isolated from the sorted subsets, and RT-qPCR was performed to study the gene expression patterns of macrophage- and DC-enriched genes (Figure 7D and Supplementary Table 2). Tyrosine-protein kinase Mer (MERTK), toll-like receptor 4 (TLR4), TLR4 coreceptor CD14, and inducible nitric oxide synthase (iNOS) were used as macrophage-enriched genes, whereas zinc finger and BTB domain-containing protein 46 (ZBTB46), C-type lectins DEC205 and DC-SIGN, chemokine receptors $\mathrm{C}-\mathrm{C}$ chemokine receptor type 6 (CCR6) and 7 (CCR7), and costimulatory receptor $C D 83$ were used as DC-enriched genes. Both subsets equally expressed MERTK $(d=0.46 ; p=$ $0.60)$, TLR4 $(d=0.42 ; p=0.62), C D 14(d=0 ; p>0.99)$, and DEC205 $(d=0.31 ; p=0.70)$. MHC-II ${ }^{\text {low }}$ cells showed slightly higher expressions of $i$ NOS $(d=0.90 ; p=0.16)$ and DC-SIGN $(d=1.32 ; p=0.08)$, whereas MHC-II ${ }^{\text {high }}$ cells showed slightly higher expressions of ZBTB46 $(d=1.48$; $p=$ $0.24)$ and CCR6 ( $d=0.58 ; p=0.05)$. More strikingly, MHC$\mathrm{II}^{\text {high }}$ cells showed much higher expressions of CCR7 $(d=$ 5.35; $p=0.02)$ and $C D 83(d=2.98 ; p=0.05)$. Thus, the differences between MHC-II low cells and MHC-II ${ }^{\text {high }}$ cells were mainly found for DC-enriched genes, especially for CCR7 and $C D 83$, which have been used by others before as maturation markers of chBMDCs $(14,30)$. In contrast, differences in gene 

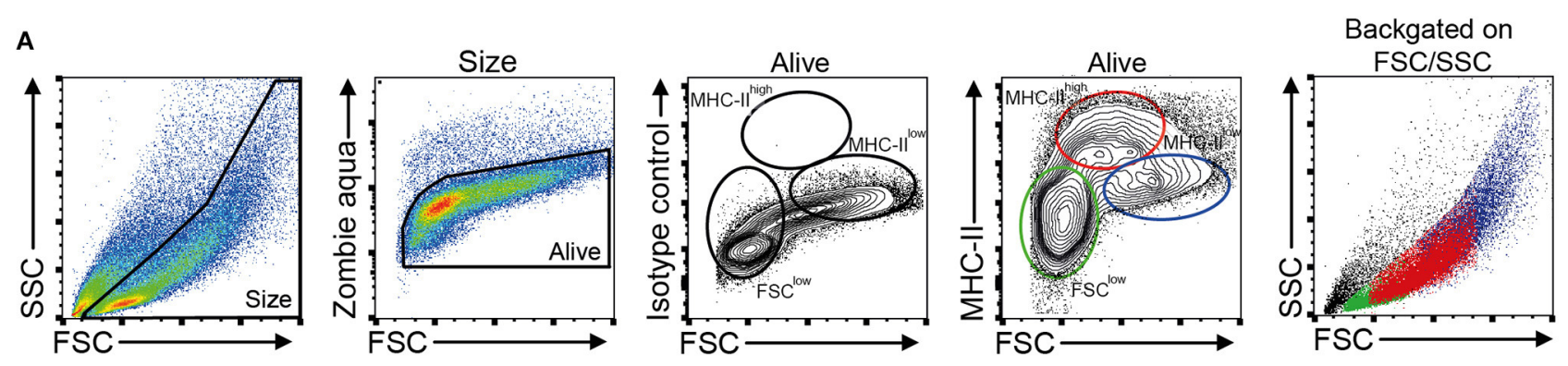

B
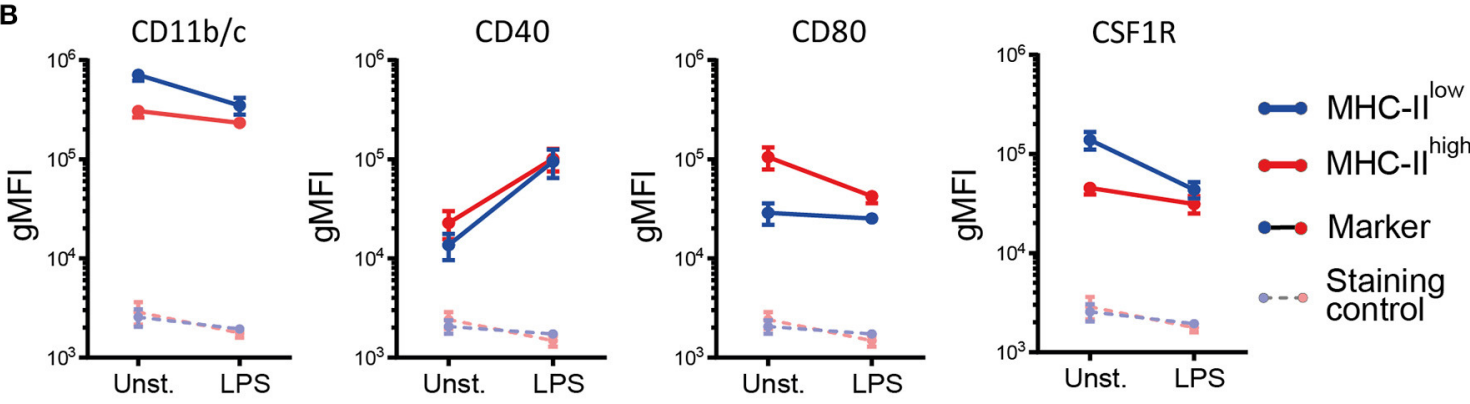

c-kit
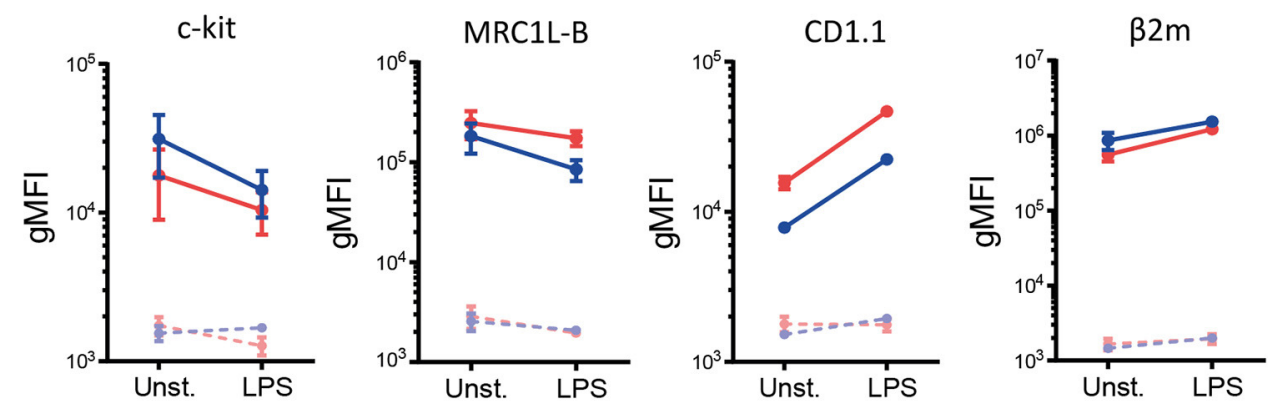

C

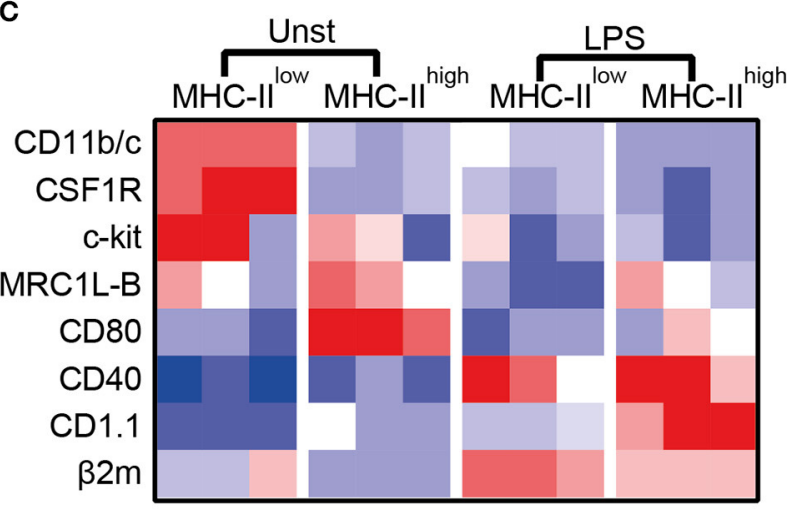

Relative

expression level:

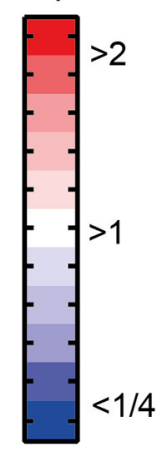

FIGURE 6 | The phenotypic distinction between major histocompatibility complex class II (MHC-II) ${ }^{\text {low }}$ and MHC-II high chicken bone marrow-derived dendritic cells (chBMDCs) is not retained after $24 \mathrm{~h}$ of lipopolysaccharide (LPS) stimulation. (A) LPS-stimulated chBMDCs were gated by a similar strategy as Figure 2A. (B) The change in myeloid marker expression upon LPS stimulation is shown for both MHC- $\|^{\text {low }}$ and MHC- $\|^{\text {high }}$ chBMDC subsets. The error bars show the SEM of three independent replicates. (C) The expression of phenotypic markers is shown in a heat-map for unstimulated and LPS-stimulated MHC-Ilow and MHC-IIigh chBMDCs. To obtain the relative expression level for each marker, the gMFI of each sample was normalized to the average gMFI of all samples.

expression were hardly found for macrophage-enriched genes. Combined with the flow cytometric data (Figure 2C) that showed higher expressions of MHC-II, CD40, and CD80 by MHCII $^{\text {high }}$ chBMDCs compared to the MHC-II low chBMDCs, the subsets seem to be DCs at different maturation states rather than different cell types.

To determine whether chBMDCs were able to induce Tcell proliferation, allogeneic mixed lymphocyte reactions were 

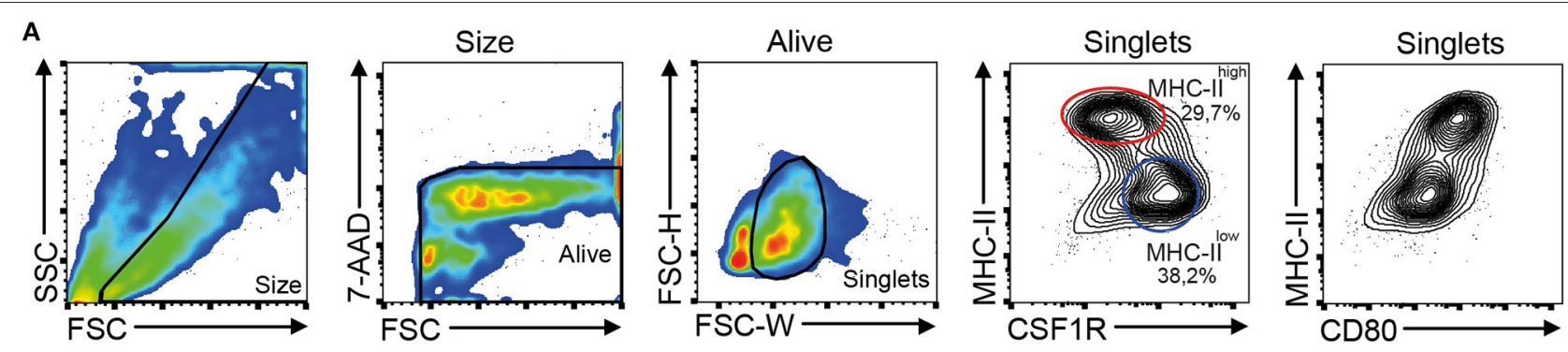

B

Sorted MHC-II ${ }^{\text {low }}$ cells
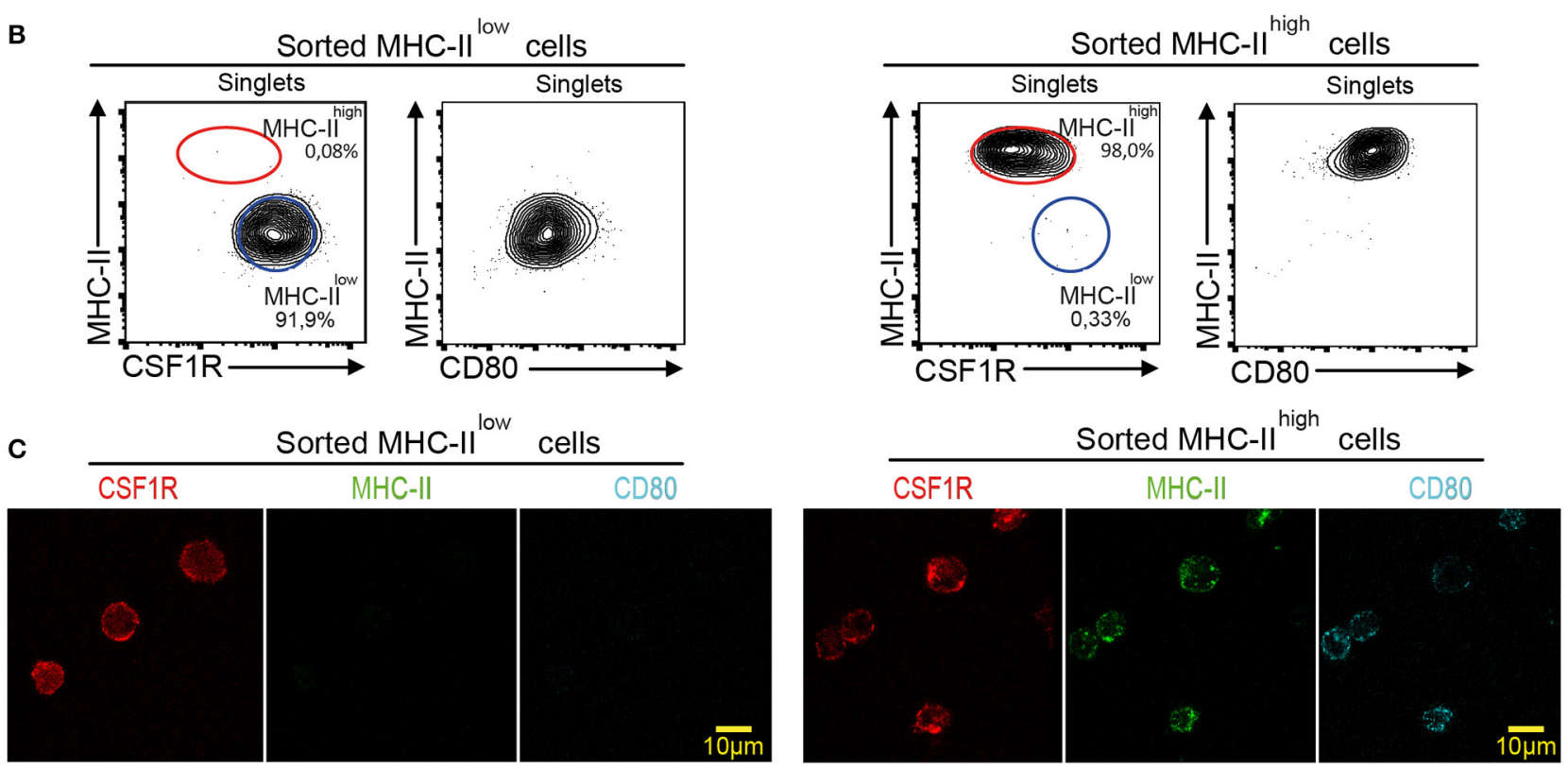

D

MERTK

TLR4

CD14

iNOS

ZBTB46
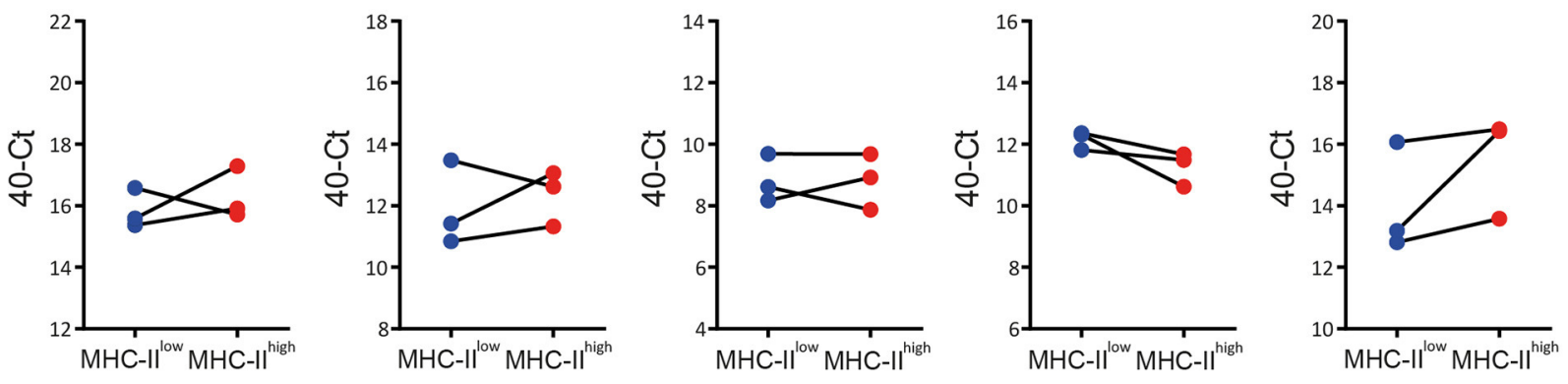

CCR6

CCR7
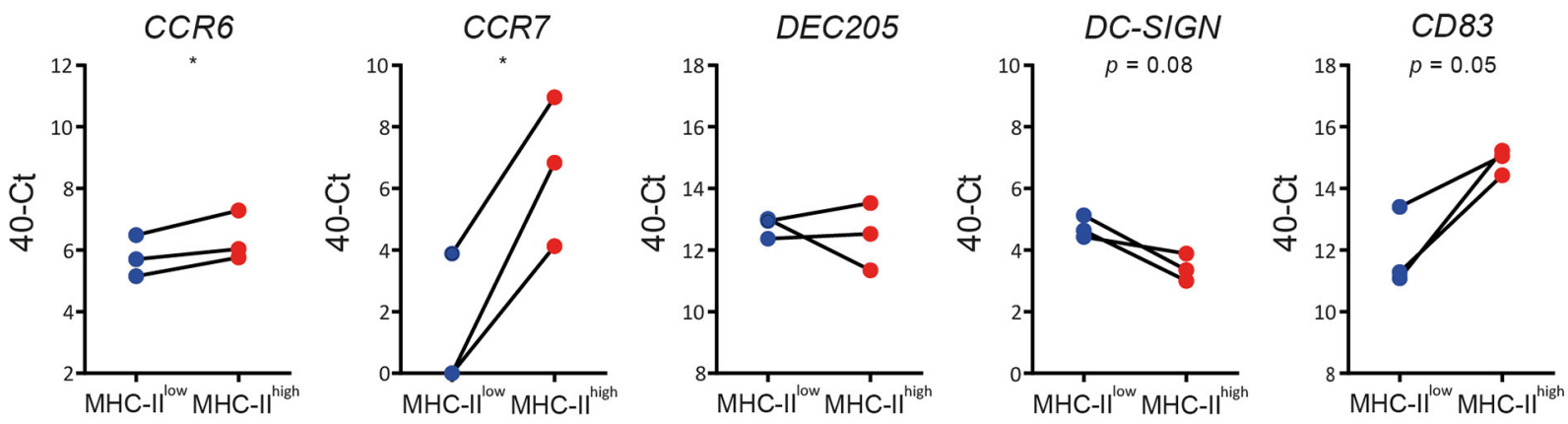

FIGURE 7 | Quantitative real-time PCR (RT-qPCR) on sorted major histocompatibility complex class II (MHC-II) ${ }^{\text {low }}$ and MHC-II ${ }^{\text {high }}$ chicken bone marrow-derived dendritic cell (chBMDC) subpopulations shows clear differential expression of DC maturation markers, but not of other DC- or macrophage-specific phenotypic 
FIGURE 7 | markers. (A) Pre-sort evaluation of chBMDCs shows the subpopulations that differ in their expression of MHC-II, CSF1R, and CD80. The subpopulations were sorted after gating for scatter profile [forward scatter (FSC)/side scatter (SSC)], viability [7-aminoactinomycin D (7-AAD)], single cells (FSC-W/FSC-H), and expression of MHC-II and colony-stimulating factor 1 receptor (CSF1R). (B) Post-sort analysis of sorted MHC-Illow and MHC-Ihigh chBMDC subpopulations shows that the sort led to populations of high purity. (C) Fluorescent microscopy shows CSF1R, MHC-II, and CD80 expressions for sorted MHC-Illow and MHC-Iligh chBMDCs. Overlays and individual channels are shown with CSF1R in red, MHC-II in green, and CD80 in cyan. (D) RT-qPCR was performed on sorted MHC-Illow and MHC-Ihigh chBMDCs. The results are expressed at the 40-Ct value with $\mathrm{Ct}$ being the number of cycles needed to reach a signal above the threshold. The results were normalized for RNA content by the average expression of housekeeping genes $28 \mathrm{~S}$ and GAPDH. Each line represents an independent replicate $(n=3)$. Statistically significant differences between chBMDC subsets are shown/indicated by ${ }^{*} p<0.05$. Non-significant $p$-values below 0.10 are given by the actual values to indicate trends close to significance.

performed with sorted chBMDC subsets. Both MHC-II ${ }^{\text {low }}$ (SI = 4.6 at 1:2 E:T ratio) and MHC-II ${ }^{\text {high }}(\mathrm{SI}=5.2$ at 1:2 E:T ratio) chBMDC subsets induced proliferation of PBMCs and did so more effectively than unsorted chBMDCs $(\mathrm{SI}=2.7)$ or PBMCs stimulated with a combination of anti-CD3, anti-CD28, and IL-2 $(\mathrm{SI}=2.0)$ (Figure 8).

\section{MHC-II high chBMDCs Become MHC-Ilow During Prolonged Incubation After Sorting} MHC-II ${ }^{\text {low }}$ and MHC-II ${ }^{\text {high }}$ chBMDCs were found to differ in the expression of DC maturation markers, at both the protein and gene expression levels, but were similar in their ability to induce PBMCs to proliferate. To gain additional proof that MHC-II ${ }^{\text {low }}$ and MHC-II ${ }^{\text {high }}$ chBMDCs represent DCs at different maturation states rather than different cell types, sorted chBMDCs were reseeded for another 1 or 3 days of cell culture and stained again for MHC-II, CSF1R, and CD80. The MHC$\mathrm{II}^{\text {low }}$ subset showed only minor changes in the expression of abovementioned markers (Figures 9A,B). In contrast, the MHCII ${ }^{\text {high }}$ subset showed higher CSF1R, lower MHC-II, and lower CD80 expression and consequently became phenotypically more similar to the MHC-II ${ }^{\text {low }}$ subset. Therefore, both chBMDC subsets seem to consist of a DC-like cell type, but these appear to be different states of maturation with MHC-II ${ }^{\text {high }}$ chBMDCs being at a more mature but reversible state. In addition, the mRNA expression levels of CCR7 and CD83 were determined and found to be decreased for both subsets after prolonged cell culture (Figure 9C).

\section{DISCUSSION}

The present study aimed to characterize GM-CSF-induced chBMDC cultures and address their heterogeneity. Despite the widespread use of in vitro grown DCs, there is still an ongoing debate about the representativeness of in vitro grown DCs for their in vivo counterparts $(20-22,24,25,31)$. In research so far, discussion focused mainly on BMDCs from mice and less on those of farm and companion animals. Early studies already described the presence of macrophage and granulocyte "contaminants" in the murine BMDC culture $(5,32)$. Moreover, murine $(20,33)$ and ovine (12) BMDC cultures, as well as bovine monocyte-derived DC cultures (34), have been shown to include CD11b ${ }^{\text {low }}$ MHC-II ${ }^{\text {high }}$ cells with a DC-like phenotype and CD11b ${ }^{\text {high }}$ MHC-II ${ }^{\text {low }}$ cells with a macrophage-like phenotype. The first study by $\mathrm{Wu}$ et al. describing the chBMDC culture already recognized its heterogeneity (14). However, this study excluded adherent and relatively small cells from analysis, which may, respectively, represent the MHC-II ${ }^{\text {low }}$ chBMDCs

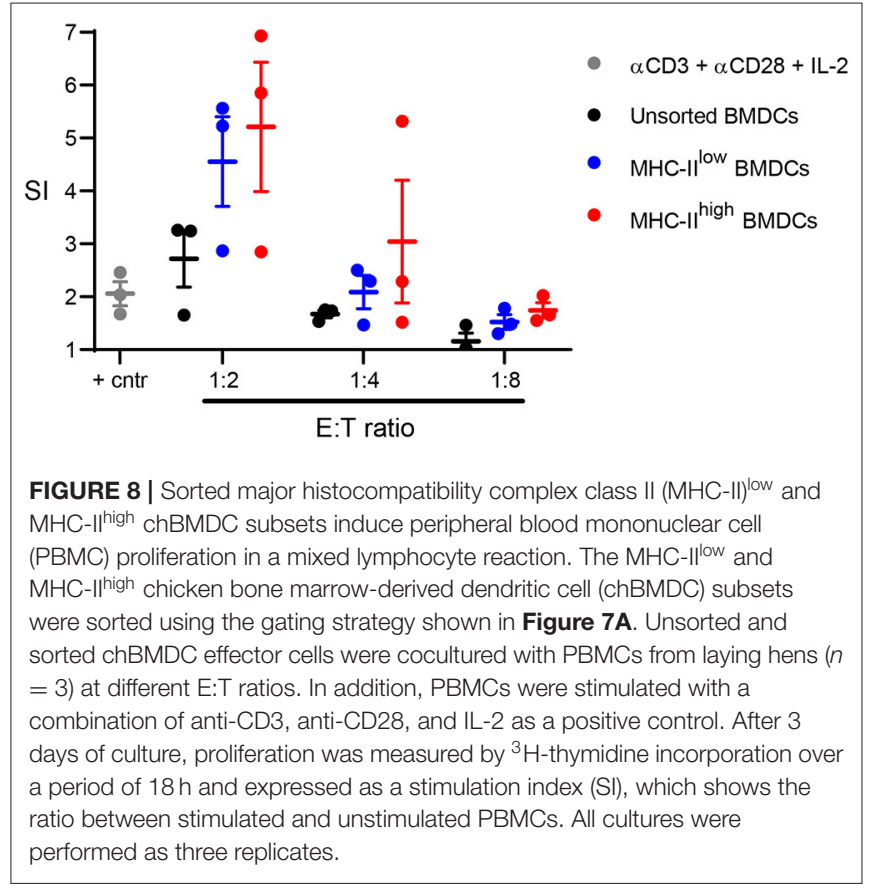

and FSC ${ }^{\text {low }}$ undifferentiated monocytes of the current study. In our opinion, these neglected cells should be characterized to interpret responses of the chBMDC culture correctly when analyzed in bulk. This has been illustrated by studies using LPSstimulated murine BMDCs, in which individual cell subsets (20) or individual cells (35) were shown to respond very differently in their maturation, cytokine expression profile, and capacity to induce T-cell proliferation. Moreover, virus infection studies with chBMDCs, including avian influenza virus $(16,26)$ and infectious bursal disease virus (36) were analyzed in bulk, while the different chBMDC subsets might vary in their susceptibility for viruses and influence the outcome of these studies.

In the present study, the chBMDC culture was found to contain MHC-II ${ }^{\text {low }}$ and MHC-II $I^{\text {high }}$ subsets, similar to BMDC and monocyte-derived DC cultures in other species $(12,20$, 33 , 34). Expression of myeloid markers, morphology, and phagocytosis capacity differed between MHC-II ${ }^{\text {low }}$ and MHCII ${ }^{\text {high }}$ subsets. Compared to the MHC-II ${ }^{\text {high }}$ subset, the MHCII $^{\text {low }}$ subset showed higher expression of CSF1R, contained larger and more granular cells based on flow cytometry scatter profile and light microscopy, and showed a higher capacity to phagocytose IgY-opsonized beads. These results suggested that the MHC-IIlow chBMDC subset consists of macrophagelike cells, in agreement with studies in murine $(20,33)$ and 


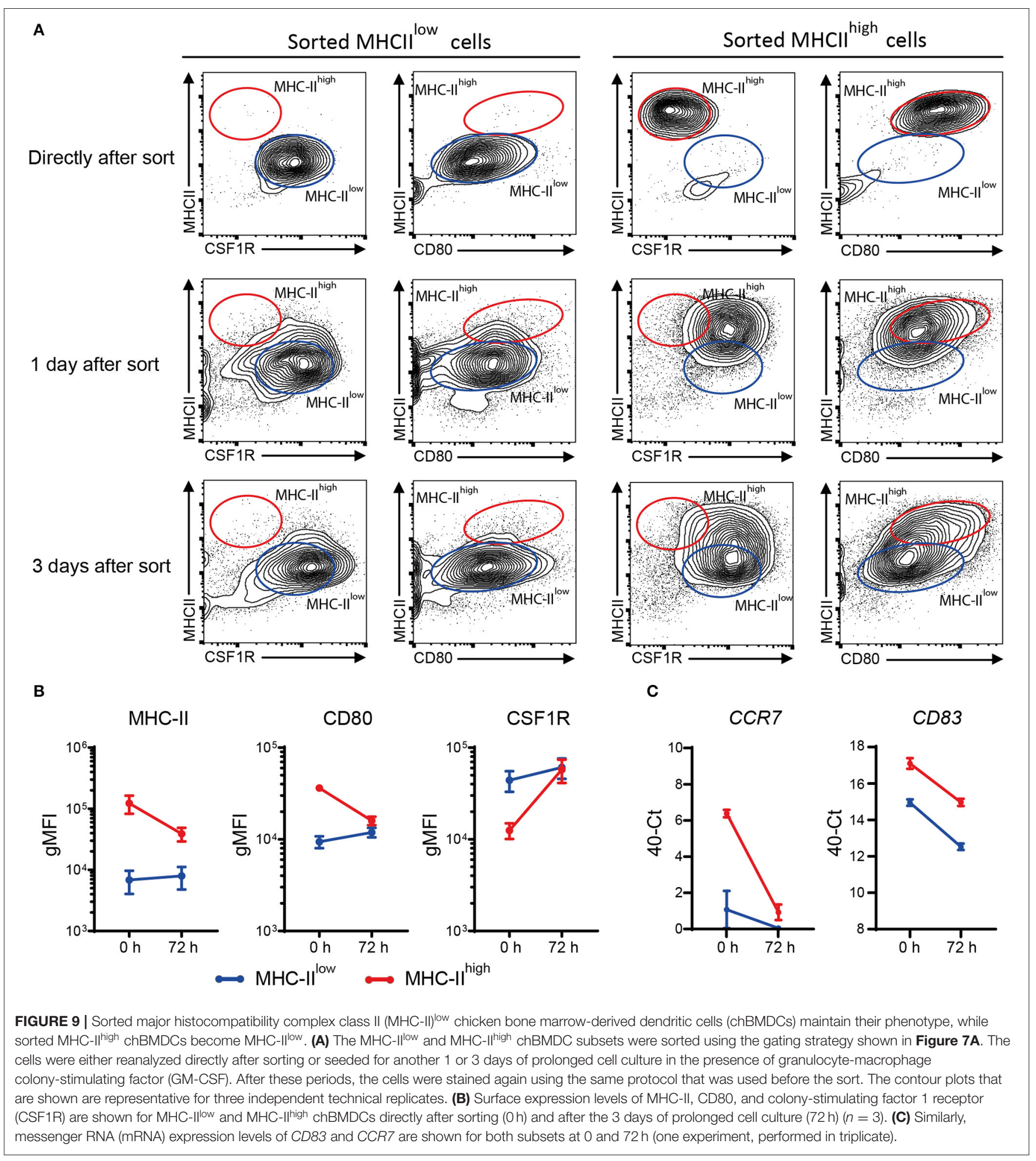

ovine (12) BMDCs, as well as bovine monocyte-derived DCs (34). In these studies, macrophage-like MHC-II ${ }^{\text {low }}$ cells showed high expression of $\mathrm{CD} 11 \mathrm{~b}$, which contributed to the distinction between the MHC-II low and MHC-II ${ }^{\text {high }}$ subsets of these BMDC cultures. Unfortunately, this was not possible for the chicken $\mathrm{CD} 11 \mathrm{~b} / \mathrm{c}$ antigen, since the corresponding antibody has not yet been confirmed to recognize either CD11b or CD11c (26). Compared to the MHC-II ${ }^{\text {low }}$ chBMDC subset, the MHC-II high subset showed higher expression of costimulatory molecules CD40 and CD80, in agreement with the DC-like phenotype shown for MHC-II ${ }^{\text {high }}$ cells in murine BMDC $(20,33)$ and bovine monocyte-derived DC cultures (34). In addition, the MHC-II high 
chBMDC subset showed a relatively high expression of the non-classical MHC molecule CD1.1, which is also indicative of a DC-like phenotype (37). Our phenotypical findings of the chBMDC subsets suggested that MHC-II low and MHC-II ${ }^{\text {high }}$ subsets, respectively, represent macrophage- and DC-like cells, in agreement with studies that used murine, ovine, and bovine in vitro DC cultures $(12,20,33,34)$. However, there is some discrepancy in the literature about murine BMDCs, since its MHC-II ${ }^{\text {low }}$ and MHC-II ${ }^{\text {high }}$ subsets have also been suggested to, respectively, represent an immature and mature phenotype of the same cell type $(3,38)$.

To further explore this alternative hypothesis, we investigated whether the chBMDC subsets were different cell types or DCs at different maturation states; MHC-II ${ }^{\text {low }}$ and MHC-II ${ }^{\text {high }}$ subsets were sorted to perform RT-qPCR for macrophage- and DCspecific markers. Both subsets showed similar expression of the macrophage-specific markers MERTK, TLR4, and CD14, which is in contrast to studies of murine BMDCs $(20,33,39)$. Moreover, no difference in expression of the DC-specific marker DEC205 was observed, while ZBTB46 and CCR6 were only moderately more highly expressed by the MHC-II high subset. The most striking differences between the chBMDC subsets were higher CD83 and CCR7 expressions and lower DC-SIGN expression for the MHC-II ${ }^{\text {high }}$ subset. Increased CD83 and CCR7 expression and decreased DC-SIGN expression have been reported as maturation signatures of human and murine monocyte-derived DCs $(20,40,41)$, suggesting that the MHC-II ${ }^{\text {high }}$ chBMDC subset represents a mature DC phenotype. Moreover, it has been shown that CCR7 expression is upregulated by chBMDCs shortly after LPS stimulation (36). Taken together, these results suggest that, rather than being different cell types, MHC$\mathrm{II}^{\text {low }}$ and MHC-II ${ }^{\text {high }}$ chBMDC subsets are DCs at different maturation states with $\mathrm{MHC}-\mathrm{II}^{\text {high }}$ chBMDC being more mature. Nonetheless, MHC-II ${ }^{\text {low }}$ and MHC-II ${ }^{\text {high }}$ chBMDCs induced similar levels of PBMC proliferation in an allogeneic mixed lymphocyte reaction. Therefore, we hypothesize that of MHCII $^{\text {high }}$ chBMDCs are in a semi-mature state, which is described in literature as the state at which DCs express high levels of MHCII and costimulatory molecules but do not produce elevated levels of pro-inflammatory cytokines or optimally stimulate $\mathrm{T}$ cell proliferation $(42,43)$. Reseeding sorted chBMDCs showed that the immature phenotype of the $\mathrm{MHC}-\mathrm{II}^{\text {low }}$ subset remained stable over time, whereas the semi-mature MHC-II ${ }^{\text {high }}$ subset decreased MHC-II and CD80 expressions and increased CSF1R expression, indicating the plasticity and reversibility of this semi-mature phenotype. The chBMDC subsets differed in phagocytosis capacity, with the MHC-II low subset being more efficient in bead uptake. This finding can also be explained by a different maturation status of the subsets, since mature DCs generally have a lower phagocytotic capacity $(44,45)$. Others observed that LPS-induced maturation diminished the phagocytosis capacity of chBMDCs (14), which was not the case for the semi-mature MHC-II ${ }^{\text {high }}$ chBMDC subset of the present study.

The semi-mature phenotype of the MHC-II ${ }^{\text {high }}$ chBMDCs must have been induced by the culture conditions that were used, since the cells were not intentionally stimulated. Since it is common practice to include IL-4 cytokine in BMDC differentiation protocols of different species, including chBMDCs (10-12, 14, 46, 47), the effect of IL-4 on the development of chBMDC subsets was investigated. When IL- 4 alone was added to the culture, we observed that few chBMDC aggregates appeared, in agreement with previous studies (17). Surprisingly, addition of IL-4 led to a lower proportion of MHC-II ${ }^{\text {high }}$ cells, inhibiting chBMDC maturation, though the number of observed chBMDC aggregates remained unaffected. To the best of our knowledge, there are no earlier reports that show an inhibitory effect of IL4 on maturation of the chBMDC culture in terms of MHC-II expression. Previous studies have only observed the occurrence of chBMDC aggregates when investigating the effects of IL-4 $(14,17)$. Studies with murine and rat BMDCs have reported that IL-4 supplementation leads to proportionally larger MHC-II high subsets $(20,47)$, in contrast to our findings for the chBMDC culture. Another study has shown that IL-4 has no effect on ovine BMDC yield or phenotype (12). Therefore, the effect of IL-4 on BMDC cultures seems to differ between species. Another parameter affecting BMDCs was the source of the serum used in the culture. FBS was found to lead to a large MHC$\mathrm{II}^{\text {high }} \mathrm{CD}^{+} 0^{+}$chBMDC population (Supplementary Figure 5), whereas chicken serum led to the immature MHC-II ${ }^{\text {low }}$ and semi-mature MHC-II ${ }^{\text {high }}$ chBMDCs of the present study.

BMDCs are often stimulated by LPS to induce maturation. In the chBMDC culture, LPS stimulation led to a striking increase in CD40 and CD1.1 expression by both chBMDC subsets, whereas expression of maturation marker CD80 by the MHC-II ${ }^{\text {high }}$ subset was unexpectedly decreased. Of note, LPS stimulation led to smaller differences between the subsets in their expression of MHC-II, CD80, and CSF1R. Overall, MHCII $^{\text {low }}$ and MHC-II ${ }^{\text {high }}$ chBMDCs responded similarly to the LPS stimulus, which favored the hypothesis that the subsets reflected one cell type at different states. In contrast, murine MHC$\mathrm{II}^{\text {low }}$ and MHC-II high BMDC subsets were shown to maintain differential gene expression profiles after LPS stimulation, which provided additional proof that the murine subsets were truly different cell types (20).

In conclusion, this study describes the heterogeneity of the GM-CSF-differentiated chBMDC culture, which comprised MHC-II ${ }^{\text {low }}$ and MHC-II ${ }^{\text {high }}$ subsets that both possess features of antigen-presenting cells. These populations were found to differ in phenotype, morphology, and their phagocytosis capacity, whereas their ability to induce PBMC proliferation was similar. Based on higher expressions of maturation markers MHCII, CD40, CD80, CD83, and CCR7 by MHC-II ${ }^{\text {high }}$ chBMDCs compared to MHC-II low chBMDCs, the MHC-II ${ }^{\text {low }}$ and MHCII $^{\text {high }}$ subsets were found to, respectively, represent immature and semi-mature chBMDCs. The semi-mature phenotype of the MHC-II ${ }^{\text {high }}$ subset was found to be reversible, since reseeding and prolonged culture of these cells led to a transition toward the immature phenotype of the MHC-II ${ }^{\text {low }}$ cells. Taken together, these results yield a thorough characterization of the chBMDC culture and explain its heterogeneity by the simultaneous presence of immature and mature subsets. Our findings are instrumental for the interpretation of experiments that use this culture in future research. 


\section{DATA AVAILABILITY STATEMENT}

All datasets generated for this study are included in the article/Supplementary Material.

\section{ETHICS STATEMENT}

The animal study was reviewed and approved by the Dutch Central Authority for Scientific Procedures on Animals.

\section{AUTHOR CONTRIBUTIONS}

$\mathrm{RB}$ and $\mathrm{CJ}$ designed the research. $\mathrm{RB}$ and GA performed the research. $\mathrm{RB}$ analyzed the data. VR, WE, and CJ supervised the work. RB, VR, WE, and CJ wrote the paper.

\section{FUNDING}

This project has received funding from the Innovative Medicines Initiative 2 Joint Undertaking under grant agreement no. 115924 (VAC2VAC). This joint undertaking receives support from

\section{REFERENCES}

1. Banchereau J, Steinman RM. Dendritic cells and the control of immunity. Nature. (1998) 392:245-52. doi: 10.1038/32588

2. Joffre O, Nolte MA, Spörri R, Sousa CRE. Inflammatory signals in dendritic cell activation and the induction of adaptive immunity. Immunol Rev. (2009) 227:234-47. doi: 10.1111/j.1600-065X.2008.00718.x

3. Gallucci S, Lolkema M, Matzinger P. Natural adjuvants: endogenous activators of dendritic cells. Nat Med. (1999) 5:1249-55. doi: 10.1038/15200

4. Medzhitov R, Janeway CA Jr. Innate immunity: impact on the adaptive immune response. Curr Opin Immunol. (1997) 9:4-9. doi: 10.1016/S0952-7915(97)80152-5

5. Inaba K, Inaba M, Romani N, Aya H, Deguchi M, Ikehara S, et al. Generation of large numbers of dendritic cells from mouse bone marrow cultures supplemented with granulocyte/macrophage colony-stimulating factor. J Exp Med. (1992) 176:1693-702. doi: 10.1084/jem.176.6.1693

6. dos Santos GG, Reinders J, Ouwehand K, Rustemeyer T, Scheper RJ, Gibbs S. Progress on the development of human in vitro dendritic cell based assays for assessment of the sensitizing potential of a compound. Toxicol Appl Pharmacol. (2009) 236:372-82. doi: 10.1016/j.taap.2009.02.004

7. Vandebriel RJ, Hoefnagel MH. Dendritic cell-based in vitro assays for vaccine immunogenicity. Hum Vaccines Immunother. (2012) 8:1323-5. doi: 10.4161/hv.21350

8. Battais F, Huppert C, Langonné I, Muller S, Sponne I. In vitro detection of chemical allergens: an optimized assay using mouse bone marrow-derived dendritic cells. Contact Dermatitis. (2017) 77:311-22. doi: 10.1111/cod.12829

9. Gutzwiller MER, Moulin HR, Zurbriggen A, Roosje P, Summerfield A. Comparative analysis of canine monocyte-and bone-marrow-derived dendritic cells. Vet Res. (2010) 41:40. doi: 10.1051/vetres/2010012

10. Bienzle D, Reggeti F, Clark ME, Chow C. Immunophenotype and functional properties of feline dendritic cells derived from blood and bone marrow. Vet Immunol Immunopathol. (2003) 96:19-30. doi: 10.1016/S0165-2427(03)00132-6

11. Hope JC, Werling D, Collins RA, Mertens B, Howard CJ. Flt-3 ligand, in combination with bovine granulocyte-macrophage colonystimulating factor and interleukin-4, promotes the growth of bovine bone marrow derived dendritic cells. Scand J Immunol. (2000) 51:60-6. doi: 10.1046/j.1365-3083.2000.00646.x

12. Foulon E, Foucras G. Two populations of ovine bone marrow-derived dendritic cells can be generated with recombinant GM-CSF and the European Union's Horizon 2020 research and innovation program and EFPIA.

\section{ACKNOWLEDGMENTS}

The authors thank Alice E.J.A.M. Sijts for her critical reading of the manuscript and Irene S. Ludwig for help with the ${ }^{3} \mathrm{H}$-thymidine incorporation assay. All fluorescent microscopy images have been acquired at the Center of Cellular Imaging, Faculty of Veterinary Medicine, Utrecht University.

\section{SUPPLEMENTARY MATERIAL}

The Supplementary Material for this article can be found online at: https://www.frontiersin.org/articles/10.3389/fimmu. 2020.00141/full\#supplementary-material

Supplementary Video 1 | This shows a 3D constructed model created by confocal microscopy from chBMDCs that phagocytosed fluorescent beads (in red) for $4 \mathrm{~h}$. The cells from the chBMDC culture were stained for MHC-II (in blue) and the plasma membrane by using WGA (in green). separated on CD11b expression. J Immunol Methods. (2008) 339:1-10. doi: 10.1016/j.jim.2008.07.012

13. Carrasco CP, Rigden RC, Schaffner R, Gerber H, Neuhaus V, Inumaru $\mathrm{S}$, et al. Porcine dendritic cells generated in vitro: morphological, phenotypic and functional properties. Immunology. (2001) 104:175-84. doi: 10.1046/j.1365-2567.2001.01299.x

14. Wu Z, Rothwell L, Young JR, Kaufman J, Butter C, Kaiser P. Generation and characterization of chicken bone marrow-derived dendritic cells. Immunology. (2010) 129:133-45. doi: 10.1111/j.1365-2567.2009.03129.x

15. De Geus ED, Tefsen B, Van Haarlem DA, Van Eden W, Van Die I, Vervelde L. Glycans from avian influenza virus are recognized by chicken dendritic cells and are targets for the humoral immune response in chicken. Mol Immunol. (2013) 56:452-62. doi: 10.1016/j.molimm.2013.06.007

16. Vervelde L, Reemers SS, van Haarlem DA, Post J, Claassen E, Rebel JM, et al Chicken dendritic cells are susceptible to highly pathogenic avian influenza viruses which induce strong cytokine responses. Dev Comp Immunol. (2013) 39:198-206. doi: 10.1016/j.dci.2012.10.011

17. Liang J, Yin Y, Qin T, Yang Q. Chicken bone marrow-derived dendritic cells maturation in response to infectious bursal disease virus. Vet Immunol Immunopathol. (2015) 164:51-5. doi: 10.1016/j.vetimm.2014. 12.012

18. Kamble NM, Jawale CV, Lee JH. Activation of chicken bone marrow-derived dendritic cells induced by a salmonella enteritidis ghost vaccine candidate. Poult Sci. (2016) 95:2274-80. doi: 10.3382/ps/pew158

19. Kamble NM, Jawale CV, Lee JH. Interaction of a live attenuated Salmonella Gallinarum vaccine candidate with chicken bone marrow-derived dendritic cells. Avian Pathol. (2016) 45:235-43. doi: 10.1080/03079457.2016.11 44919

20. Helft J, Böttcher J, Chakravarty P, Zelenay S, Huotari J, Schraml BU, et al. GM-CSF mouse bone marrow cultures comprise a heterogeneous population of CD11c+ MHCII+ macrophages and dendritic cells. Immunity. (2015) 42:1197-211. doi: 10.1016/j.immuni.2015.05.018

21. Lutz $\mathrm{MB}$, Inaba $\mathrm{K}$, Schuler $\mathrm{G}$, Romani N. Still alive and kicking: in-vitro-generated GM-CSF dendritic cells!. Immunity. (2016) 44:1-2. doi: 10.1016/j.immuni.2015.12.013

22. Guilliams M, Malissen B. A death notice for in-vitro generated GM-CSF dendritic cells? Immunity. (2015) 42:988-90. doi: 10.1016/j.immuni.2015.05.020

23. Guilliams M, Dutertre CA, Scott CL, McGovern N, Sichien D, Chakarov S, et al. Unsupervised high-dimensional analysis aligns 
dendritic cells across tissues and species. Immunity. (2016) 45:669-84. doi: 10.1016/j.immuni.2016.08.015

24. Guilliams M, Ginhoux F, Jakubzick C, Naik SH, Onai N, Schraml BU, et al. Dendritic cells, monocytes and macrophages: a unified nomenclature based on ontogeny. Nat Rev Immunol. (2014) 14:571-8. doi: 10.1038/nri3712

25. Helft J, Böttcher JP, Chakravarty P, Zelenay S, Huotari J, Schraml BU, et al. Alive but confused: heterogeneity of CD11c+ MHC class II+ cells in GM-CSF mouse bone marrow cultures. Immunity. (2016) 44:3-4. doi: 10.1016/j.immuni.2015.12.014

26. De Geus ED, Jansen CA, Vervelde L. Uptake of particulate antigens in a nonmammalian lung: phenotypic and functional characterization of avian respiratory phagocytes using bacterial or viral antigens. J Immunol. (2012) 188:4516-26. doi: 10.4049/jimmunol.1200092

27. Avery S, Rothwell L, Degen WD, Schijns VE, Young J, Kaufman J, et al. Characterization of the first nonmammalian T2 cytokine gene cluster: the cluster contains functional single-copy genes for IL-3, IL-4, IL-13, and GMCSF, a gene for IL-5 that appears to be a pseudogene, and a gene encoding another cytokinelike transcript, KK34. J Interferon Cytokine Res. (2004) 24:600-10. doi: $10.1089 /$ jir.2004.24.600

28. Schindelin J, Arganda-Carreras I, Frise E, Kaynig V, Longair M, Pietzsch T, et al. Fiji: an open-source platform for biological-image analysis. Nat Methods. (2012) 9:676-82. doi: 10.1038/nmeth.2019

29. Eldaghayes I, Rothwell L, Williams A, Withers D, Balu S, Davison F, et al. Infectious bursal disease virus: strains that differ in virulence differentially modulate the innate immune response to infection in the chicken bursa. Viral Immunol. (2006) 19:83-91. doi: 10.1089/vim.2006.19.83

30. Wu Z, Hu T, Kaiser P. Chicken CCR6 and CCR7 are markers for immature and mature dendritic cells respectively. Dev Comp Immunol. (2011) 35:563-7. doi: 10.1016/j.dci.2010.12.015

31. Guilliams M, Malissen B. A matter of perspective: moving from a preomic to a systems-biology vantage of monocyte-derived cell function and nomenclature. Immunity. (2016) 44:5-6. doi: 10.1016/j.immuni.2015.12.020

32. Lutz MB, Kukutsch N, Ogilvie AL, Rößner S, Koch F, Romani N, et al. An advanced culture method for generating large quantities of highly pure dendritic cells from mouse bone marrow. J Immunol Methods. (1999) 223:7792. doi: 10.1016/S0022-1759(98)00204-X

33. Na YR, Jung D, Gu GJ, Seok SH. GM-CSF grown bone marrow derived cells are composed of phenotypically different dendritic cells and macrophages. Mol Cells. (2016) 39:734. doi: 10.14348/molcells.2016.0160

34. Guzman E, Pujol ME, Ribeca P, Montoya M. Bovine derived in vitro cultures generate heterogeneous populations of antigen presenting cells. Front Immunol. (2019) 10:612. doi: 10.3389/fimmu.2019.00612

35. Shalek AK, Satija R, Adiconis X, Gertner RS, Gaublomme JT, Raychowdhury R, et al. Single-cell transcriptomics reveals bimodality in expression and splicing in immune cells. Nature. (2013) 498:236-40. doi: 10.1038/nature12172

36. Yasmin AR, Yeap SK, Tan SW, Hair-Bejo M, Fakurazi S, Kaiser P, et al. In vitro characterization of chicken bone marrow-derived dendritic cells following infection with very virulent infectious bursal disease virus. Avian Pathol. (2015) 44:452-62. doi: 10.1080/03079457.2015.1084997

37. Cao X, Sugita M, van der Wel N, Lai J, Rogers RA, Peters PJ, et al. CD1 molecules efficiently present antigen in immature dendritic cells and traffic independently of MHC class II during dendritic cell maturation. J Immunol. (2002) 169:4770-7. doi: 10.4049/jimmunol.169.9.4770
38. Jiang A, Bloom O, Ono S, Cui W, Unternaehrer J, Jiang S, et al. Disruption of E-cadherin-mediated adhesion induces a functionally distinct pathway of dendritic cell maturation. Immunity. (2007) 27:610-24. doi: 10.1016/j.immuni.2007.08.015

39. Satpathy AT, Kc W, Albring JC, Edelson BT, Kretzer NM, Bhattacharya D, et al. Zbtb46 expression distinguishes classical dendritic cells and their committed progenitors from other immune lineages. J Exp Med. (2012) 209:1135-52. doi: 10.1084/jem.20120030

40. Sennikov SV, Falaleeva SA, Shkaruba NS, Chumasova OA, Obleukhova IA, Sizikov AE, et al. Maturation and cytokine production potential of dendritic cells isolated from rheumatoid arthritis patients peripheral blood and induced in vitro. Hum Immunol. (2016) 77:930-6. doi: 10.1016/j.humimm.2016.07.005

41. Geijtenbeek TB, Torensma R, van Vliet SJ, van Duijnhoven GC, Adema GJ, van Kooyk Y, et al. Identification of DC-SIGN, a novel dendritic cellspecific ICAM-3 receptor that supports primary immune responses. Cell. (2000) 100:575-85. doi: 10.1016/S0092-8674(00)80693-5

42. Lutz MB, Schuler G. Immature, semi-mature and fully mature dendritic cells: which signals induce tolerance or immunity? Trends Immunol. (2002) 23:445-9. doi: 10.1016/S1471-4906(02)02281-0

43. Dudek AM, Martin S, Garg AD, Agostinis P. Immature, semi-mature, and fully mature dendritic cells: toward a DC-cancer cells interface that augments anticancer immunity. Front Immunol. (2013) 4:438. doi: $10.3389 /$ fimmu.2013.00438

44. $\mathrm{Wu} \mathrm{Z}$, Kaiser $\mathrm{P}$. Antigen presenting cells in a non-mammalian model system, the chicken. Immunobiology. (2011) 216:1177-83. doi: 10.1016/j.imbio.2011.05.012

45. Platt CD, Ma JK, Chalouni C, Ebersold M, Bou-Reslan H, Carano RA, et al. Mature dendritic cells use endocytic receptors to capture and present antigens. Proc Natl Acad Sci USA. (2010) 107:4287-92. doi: 10.1073/pnas.0910 609107

46. Menges M, Baumeister T, Rössner S, Stoitzner P, Romani N, Gessner A, et al. IL-4 supports the generation of a dendritic cell subset from murine bone marrow with altered endocytosis capacity. J Leukoc Biol. (2005) 77:535-43. doi: $10.1189 / \mathrm{jlb} .0804473$

47. Wang S, Sun X, Zhou H, Zhu Z, Zhao W, Zhu C. Interleukin-4 affects the mature phenotype and function of rat bone marrow-derived dendritic cells. Mol Med Rep. (2015) 12:233-7. doi: 10.3892/mmr.2015.3349

Disclaimer: The contents of this article represent the authors' scientific conclusions, and neither IMI nor the European Union, EFPIA, or any associated partners are responsible for any use that may be made of the information contained therein.

Conflict of Interest: The authors declare that the research was conducted in the absence of any commercial or financial relationships that could be construed as a potential conflict of interest.

Copyright (๑) 2020 van den Biggelaar, Arkesteijn, Rutten, van Eden and Jansen. This is an open-access article distributed under the terms of the Creative Commons Attribution License (CC BY). The use, distribution or reproduction in other forums is permitted, provided the original author(s) and the copyright owner(s) are credited and that the original publication in this journal is cited, in accordance with accepted academic practice. No use, distribution or reproduction is permitted which does not comply with these terms. 